\title{
CERTIFIED REDUCED BASIS METHODS AND OUTPUT BOUNDS FOR THE HARMONIC MAXWELL'S EQUATIONS
}

\author{
YANLAI CHEN ${ }^{\dagger}$, JAN S. HESTHAVEN ${ }^{\dagger}$, YVON MADAY Y $^{\ddagger}$, AND JERÓNIMO \\ RODRÍGUEZ §
}

\begin{abstract}
We propose certified reduced basis methods (RBM) for the efficient and reliable evaluation of a general output that is implicitly connected to a given parametrized input through the harmonic Maxwell's equations. The truth approximation and the development of the reduced basis through a greedy approach is based on a discontinuous Galerkin approximation of the linear partial differential equation. The formulation allows the use of different approximation spaces for solving the primal and the dual truth approximation problems to respect the characteristics of both problem types, leading to an overall reduction in the off-line computational effort.

The main features of the method are: i) rapid convergence on the entire representative set of parameters, ii) rigorous a posteriori error estimators for the output and iii) a parameter independent off-line phase and a computationally very efficient on-line phase to enable the rapid solution of manyquery problems arising in control, optimization, and design. The versatility and performance of this approach is shown through a numerical experiment, illustrating the modeling of material variations and problems with resonant behavior.
\end{abstract}

Key words. Reduced basis methods; a priori theory; a posteriori error estimation; discontinuous Galerkin methods; Maxwell's equations.

AMS subject classifications. $65 \mathrm{~N} 15,65 \mathrm{~N} 30,78 \mathrm{~A} 25$

1. Introduction. Many applications related to computational optimization, control and design, require the ability to rapidly, perhaps even in real time, and accurately predict some quantities of interest under the variation of a set of parameters. A similar need is found in the development of large simulation based databases or the development of efficient ways to quantify uncertainty and its impact.

In such cases, an output of interest, here denoted by $s^{e}$, is often provided by a functional applied to the solution of a parametrized partial differential equation (PDE) that describes the underlying problem. More precisely,

$$
\begin{gathered}
\text { For an input } \nu \in \mathcal{D} \subset \mathbb{R}^{q} \text { the output is defined by } \\
s^{e}(\nu):=l\left(u^{e}(\nu) ; \nu\right) \in \mathbb{C}, \\
\text { where } u^{e}(\nu) \in X^{e} \text { is the solution of the linear PDE } \\
\mathcal{L}(\nu) u^{e}(\nu)=f(\nu) .
\end{gathered}
$$

The $q$-dimensional set of parameters $\nu$, here denoting the input, determines a particular configuration of the system. In practice, the parameters can be related to the description of sources, materials, geometries, uncertainties etc. We have an implicit relationship between the input and the output through the partial differential equation.

Our primary goal is to develop a systematic approach to obtain an accurate and reliable approximation of the output of interest at very low computational cost for

\footnotetext{
${ }^{\dagger}$ Division of Applied Mathematics, Brown University, 182 George Street, Providence, RI 02912

‡Université Pierre et Marie Curie-Paris6, UMR 7598, Laboratoire J.-L. Lions, Paris, F-75005 France

§Departamento de Matemática Aplicada, Facultade de Matemáticas, USC, 15782 Santiago de Compostela, A Coruña, Spain

TE-mail addresses: Yanlai_Chen@Brown.edu (Y.Chen) Jan.Hesthaven@Brown.edu (J.Hesthaven) maday@ann.jussieu.fr (Y.Maday) jeronimo.rodriguez@usc.es (J.Rodríguez)
} 
applications where many queries, i.e., solutions, are needed. We will explore the use of a reduced basis method by recognizing, and implicitly assuming, that the parameter dependent solution $u^{e}(\nu)$ is not simply an arbitrary member of the infinite-dimensional space associated with the partial differential equation, but rather that it evolves on a lower-dimensional manifold induced by the parametric dependence. Under this assumption we can expect that as $\nu\left(\in \mathcal{D} \subset \mathbb{R}^{q}\right)$ varies, the set of all solutions $u^{e}(\nu)$ can be well approximated by a finite and low dimensional vector space. Hence, for a well chosen set of $N$ parameters $\nu_{i}$, there exist coefficients $c_{i}=c_{i}^{N}(\nu)$ for any $\nu \in \mathcal{D}$ such that $\sum_{i=1}^{N} c_{i} u^{e}\left(\nu_{i}\right)$ is very close to $u^{e}(\nu)$ when measured in an appropriate norm. The reduced basis method was first introduced in the 1970's for nonlinear structural analysis [1, 27] and it was subsequently abstracted, analyzed [5, 32] and generalized to other types of parametrized partial differential equations $[12,28]$. Most of these earlier works focus on arguments that are local in the parameter space. Expansions to a low dimensional manifold are typically defined around a particular point of interest and the associated a priori analysis relies on asymptotic arguments on sufficiently small neighborhoods $[8,30]$. In such cases, the computational improvements are quite modest. In $[3,17]$ a global approximation space was built by using solutions of the governing PDE at globally sampled points in the parameter space, resulting in a vastly improved method. However, no a priori theory or a posteriori error estimators were developed in this early work.

In recent years, a number of novel ideas and essential new features have been presented $[22,21,39,31,38,4,10,37,34]$. In particular, global approximation spaces have been introduced and uniform exponential convergence of the reduced basis approximation has been numerically observed and confirmed in [23] where the first theoretical a priori convergence result for a one dimensional parametric space problem is presented. The development of rigorous a posteriori error estimators have also been presented, thereby transforming the reduced basis methods from an experimental technique to a computational approach with a true predictive value.

Furthermore, in cases where the problem satisfies an affine assumption; that is, the operators and the data can be written as a linear combination of functions with separable dependence of the parameter and the spatial variation of the data, an off-line/online computational strategy can be formulated. The off-line part of the algorithm, consisting of the generation of the reduced basis space, is $\nu$-independent and can be done in preprocessing. The computational cost of the on-line part depends solely on the dimension of the reduced basis space and the parametric complexity of the problem, while the dependence on the complexity of the truth approximation has been removed, resulting in a highly efficient approach.

When the data of the PDE are not affine, this computational strategy can not be directly applied anymore and the on-line computational cost of the algorithm may be rather high. Recently, in [4], a procedure allowing the treatment of some of these nonaffine operators has been presented and shown to recover the off-line/on-line efficiency of the original algorithm. This technique, which also provides asymptotic a posteriori error estimators, has been successfully used in several applications [10, 9, 26, 36]. Following standard techniques, we will consider (1.1) in weak form as

For an input $\nu \in \mathcal{D} \subset \mathbb{R}^{p}$ the output is defined by

$$
s^{e}(\nu):=l\left(u^{e}(\nu) ; \nu\right) \in \mathbb{C},
$$

where $u^{e}(\nu) \in X^{e}$ is the exact solution of the linear PDE

$$
a\left(u^{e}(\nu), v ; \nu\right)=f(v ; \nu), \quad \forall v \in X^{e} .
$$


In contrast to most previous work, we focus on electromagnetic wave propagation problems. There are many applications demanding rapid and reliable solutions to these problems, e.g. radar cross section prediction [20] and waveguide design [11]. We remark that RBM has been successfully applied to similar non-coercive problems such as the Helmholtz equation for time-harmonic acoustics [36, 39]. It was also used to achieve fast optimization of electrostatic and magnetostatic problems such as cogging torque minimization $[19,18]$.

It is useful to realize that the output $s^{e}(\nu)$ can also be obtained using adjoint techniques. Consider the adjoint problem

$$
\mid \begin{aligned}
& \text { Seek } \psi^{e}(\nu) \in X^{e} \text { such that } \\
& a\left(\phi, \psi^{e}(\nu) ; \nu\right)=l(\phi ; \nu), \quad \forall \phi \in X^{e} .
\end{aligned}
$$

We will refer to (1.2) as the primal problem, and to (1.3) as the dual problem. One has

$$
f\left(\psi^{e}(\nu) ; \nu\right)=a\left(u^{e}(\nu), \psi^{e}(\nu) ; \nu\right)=l\left(u^{e}(\nu) ; \nu\right)=s^{e}(\nu) .
$$

As we will discuss later (see also [29, 31] for more details), an efficient and accurate way to compute the output needs to solve both problems, the nature of which might be slightly different except in the simple self-adjoint case. For this reason we will allow the use of different approximation spaces for solving these two problems. This yields some additional flexibility and may reduce the off-line computational effort without adversely impacting the accuracy.

To solve the primal and dual problems for specific parameter choices, we will use a discontinuous Galerkin method [13]. These methods have developed rapidly during the last decade and have proven themselves to be an efficient and accurate way to solve general wave problems and Maxwell's equations in particular. While the analysis of the resulting reduced basis method is influenced somewhat by the choice of the approximation technique for the primal/dual problems, the general framework developed here can be expected to generalize to other techniques such as classic finite element methods.

The paper is organized as follows. In Sec. 2 we briefly outline the harmonic Maxwell's equations and discuss appropriate boundary conditions and solution spaces. This sets the stage for Sec. 3 which discusses the discontinuous Galerkin method for discretizing Maxwell's equations. Section 4 is the first main part of this work and outlines in detail the development of the reduced basis technique, including a priori theory and a posteriori error estimates. In Sec. 5 we address the second main topic related to the algorithmic aspects of the method while Sec. 6 is devoted to illustrating the performance of the algorithm on a non-trivial test case. Section 7 contains a few concluding remarks and suggestions for future work.

2. The harmonic Maxwell's equations. Let us consider the harmonic chargefree Maxwell's equations defined on $\boldsymbol{x} \in \Omega \subset \mathbb{R}^{3}$ with $\partial \Omega$ representing the boundary of the domain of interest.

$$
\begin{aligned}
& i \omega \varepsilon \boldsymbol{E}=\nabla \times \boldsymbol{H}+\boldsymbol{J}, \quad i \omega \mu \boldsymbol{H}=-\nabla \times \boldsymbol{E} \\
& \nabla \cdot(\varepsilon \boldsymbol{E})=0, \quad \nabla \cdot(\mu \boldsymbol{H})=0 .
\end{aligned}
$$

Here $\boldsymbol{E}(\boldsymbol{x})=\left(E_{x}, E_{y}, E_{z}\right), \boldsymbol{H}(\boldsymbol{x})=\left(H_{x}, H_{y}, H_{z}\right)$ represent the electric and magnetic vectors phasor fields, $\boldsymbol{J}(\boldsymbol{x})=\left(J_{x}, J_{y}, J_{z}\right)$ the current source, and $(\varepsilon(\boldsymbol{x}), \mu(\boldsymbol{x}))$ are the 
tensors of electric permittivity and magnetic permeability, respectively. To simplify matters we assume isotropic materials in which case $\varepsilon(\boldsymbol{x})=\varepsilon(\boldsymbol{x}) \mathrm{I}$ where $\varepsilon(\boldsymbol{x})$ is a scalar and I a 3-identity matrix. We make similar assumption for the permeability. The parameter $\omega$ reflects the angular frequency of the electromagnetic wave.

The boundary conditions on the electric field are imposed on the tangential components, $\boldsymbol{n} \times \boldsymbol{E}$, which must remain continuous across a material interface endowed with the outward pointing normal vector, $\boldsymbol{n}$. The tangential field vanishes along a perfectly electrically conducting metallic wall. For the magnetic field, the tangential components $\boldsymbol{n} \times \boldsymbol{H}$ likewise remains continuous across material interfaces while at a perfectly electrically conducting wall, $\boldsymbol{n} \cdot \boldsymbol{H}$ vanishes.

The natural space for solutions to Maxwell's equations with vanishing tangential component for the electric field is $X^{e}=H_{0}(\operatorname{curl} ; \Omega)$, defined as

$$
H_{0}(\operatorname{curl} ; \Omega)=\left\{\boldsymbol{v} \in\left(L^{2}(\Omega)\right)^{3} \mid \nabla \times \boldsymbol{v} \in\left(L^{2}(\Omega)\right)^{3}, \boldsymbol{n} \times \boldsymbol{v}=0 \text { on } \partial \Omega\right\} .
$$

If we define the standard $L^{2}$-inner product and norm as

$$
(\boldsymbol{u}, \boldsymbol{v})_{\Omega}=\int_{\Omega} \boldsymbol{u} \cdot \boldsymbol{v}^{*} d \boldsymbol{x},\|\boldsymbol{u}\|^{2}=(\boldsymbol{u}, \boldsymbol{u})_{\Omega},
$$

the natural norm associated with Maxwell's equations is

$$
\|\boldsymbol{v}\|_{H(\text { curl })}^{2}=\|\boldsymbol{v}\|^{2}+\|\nabla \times \boldsymbol{v}\|^{2} .
$$

The problem can be further simplified to recover the second order curl-curl formulation

$$
\nabla \times \mu(\boldsymbol{x})^{-1} \nabla \times \boldsymbol{E}-\varepsilon(\boldsymbol{x}) \omega^{2} \boldsymbol{E}=i \omega \boldsymbol{J}=\boldsymbol{f}, \quad \boldsymbol{x} \in \Omega .
$$

Naturally, an equivalent equation can be obtained for the magnetic field.

If we define the bilinear form $a(\boldsymbol{u}, \boldsymbol{v}): X^{e} \times X^{e} \rightarrow \mathbb{C}$ as

$$
a(\boldsymbol{u}, \boldsymbol{v})=\left(\mu^{-1} \nabla \times \boldsymbol{u}, \nabla \times \boldsymbol{v}\right)_{\Omega}-\omega^{2}(\varepsilon \boldsymbol{u}, \boldsymbol{v})_{\Omega}, \quad \forall \boldsymbol{u}, \boldsymbol{v} \in X^{e},
$$

then the variational statement for the curl-curl equation is: find $\boldsymbol{E} \in X^{e}$ such that

$$
a(\boldsymbol{E}, \boldsymbol{v})=f(\boldsymbol{v})
$$

provided we consider the simple case of perfectly electrically conducting walls for $\boldsymbol{x} \in \partial \Omega$. Here,

$$
f(\boldsymbol{v})=(\boldsymbol{f}, \boldsymbol{v})_{\Omega}=\int_{\Omega} \boldsymbol{f} \cdot \boldsymbol{v}^{*} d \boldsymbol{x}, \forall \boldsymbol{v} \in X^{e},
$$

More general situations and the general question of well-posedness of Maxwell's equations are discussed at length in [25].

The dual problem for this case is: find $\psi \in X^{e}$ such that

$$
a(\phi, \psi)=\ell(\phi) \forall \phi \in X^{e},
$$

where,

$$
\ell(\phi)=(\phi, l)_{\Omega}
$$


3. The discontinuous Galerkin approximation. To solve (2.2), we employ a discontinuous Galerkin method [13], see also [14, 15]. To get started, let $\left\{\mathrm{D}^{k}: k=1, \ldots, K\right\}$ be a collection of disjoint elements that partition $\Omega$. The triangulation needs not be conforming. On each of these elements, we assume that we can approximate the solution of $(2.2), \boldsymbol{E} \in X^{e}$, by a $p$ th order polynomial; that is we assume

$$
\boldsymbol{x} \in \mathrm{D}^{k}: \quad \boldsymbol{E} \simeq \boldsymbol{E}_{h}^{k} \in\left[\mathrm{P}_{p}^{k}(\boldsymbol{x})\right]^{3},
$$

where $\mathrm{P}_{p}^{k}$ is the space of $p$ th order complex-valued polynomials defined on $\mathrm{D}^{k}$. The global space of solutions, $X^{e}$, is thus approximated by $X_{h}^{e}=\oplus_{k}\left[\mathrm{P}_{p}^{k}\right]^{3}$ and we seek $\left(\boldsymbol{q}_{h}, \boldsymbol{E}_{h}\right)$ that satisfy the following elementwise statement

$$
\begin{gathered}
\left(\boldsymbol{q}_{h}, \nabla \times \boldsymbol{v}_{h}\right)_{\mathrm{D}^{k}}-\omega^{2}\left(\varepsilon \boldsymbol{E}_{h}, \boldsymbol{v}_{h}\right)_{\mathrm{D}^{k}}+\left(\boldsymbol{n} \times \widehat{\boldsymbol{q}_{h}}, \boldsymbol{v}_{h}\right)_{\partial \mathrm{D}^{k}}=\left(\boldsymbol{f}_{h}, \boldsymbol{v}_{h}\right)_{\mathrm{D}^{k}}, \forall \boldsymbol{v}_{h} \in X_{h}^{e}, \\
\left(\mu \boldsymbol{q}_{h}, \boldsymbol{w}_{h}\right)_{\mathrm{D}^{k}}=\left(\boldsymbol{E}_{h}, \nabla \times \boldsymbol{w}_{h}\right)_{\mathrm{D}^{k}}+\left(\boldsymbol{n} \times \widehat{\boldsymbol{E}_{h}}, \boldsymbol{w}_{h}\right)_{\partial \mathrm{D}^{k}}, \forall \boldsymbol{w}_{h} \in X_{h}^{e},
\end{gathered}
$$

where we denote the piecewise polynomial representation of $\boldsymbol{f}$ by $\boldsymbol{f}_{h}$ and $\boldsymbol{n}$ is the outward unit normal vector to the element $D^{k}$. In these last equations, we have introduced the numerical fluxes $\widehat{\boldsymbol{q}_{h}}$, and $\widehat{\boldsymbol{E}_{h}}$, which enforce connectivity between the elements and give stability of the discrete problem.

Next, we obtain discrete version of the formulations given by (2.4) and (2.3) and reduce the problem of finding $\boldsymbol{E}_{h} \in X_{h}^{e}$ to be that given as

$$
a_{h}\left(\boldsymbol{E}_{h}, \boldsymbol{v}_{h}\right)=f_{h}\left(\boldsymbol{v}_{h}\right), \quad \forall \boldsymbol{v}_{h} \in X_{h}^{e} .
$$

To do so, let us introduce some notations. $\mathcal{F}^{o}$ denotes the set of all interior faces $e=\partial D^{+} \cap \partial D^{-}$for some two elements $D^{+}$and $D^{-}$, and $\mathcal{F}^{\partial}$ the set of all boundary faces $e=\partial D \cap \partial \Omega$ for some element $D$. We set $\mathcal{F}=\mathcal{F}^{o} \cup \mathcal{F}^{\partial}$. For a given internal face $e=\partial D^{+} \cap \partial D^{-}$, we define the outward unit normal vector to $D^{+}$(resp. $D^{-}$) by $\boldsymbol{n}^{+}$(resp. $\boldsymbol{n}^{-}$). Let $\boldsymbol{u}$ be a vector field. With $\boldsymbol{u}^{ \pm}:=\boldsymbol{u}_{\mid \partial D^{ \pm}}$, we define the average and the tangential jump of $\boldsymbol{u}$ in the standard way

$$
\{\{\boldsymbol{u}\}\}=\left\{\begin{array}{ll}
\frac{\boldsymbol{u}^{-}+\boldsymbol{u}^{+}}{2}, & \text { on } \mathcal{F}^{o}, \\
\boldsymbol{u}, & \text { on } \mathcal{F}^{\partial},
\end{array} \quad \llbracket \boldsymbol{u} \rrbracket_{T}= \begin{cases}\boldsymbol{n}^{-} \times \boldsymbol{u}^{-}+\boldsymbol{n}^{+} \times \boldsymbol{u}^{+}, & \text {on } \mathcal{F}^{o}, \\
\boldsymbol{n} \times \boldsymbol{u}, & \text { on } \mathcal{F}^{\partial} .\end{cases}\right.
$$

We also introduce the $L^{2}$-inner product on any set of faces $\widetilde{\mathcal{F}} \subset \mathcal{F}$ by

$$
(\varphi, \psi)_{\widetilde{\mathcal{F}}}=\sum_{e \in \widetilde{\mathcal{F}}} \int_{e} \varphi \cdot \psi^{*} d \boldsymbol{\gamma}
$$

where $\varphi$ and $\boldsymbol{\psi}$ are the average or tangential jump defined above. Adding (3.1) over all the elements, we obtain the following global formulation $\left(\nabla_{h} \times\right.$ denotes the local curl-operator),

$$
\begin{aligned}
&\left(\boldsymbol{q}_{h}, \nabla_{h} \times \boldsymbol{v}_{h}\right)_{\Omega}-\omega^{2}\left(\varepsilon \boldsymbol{E}_{h}, \boldsymbol{v}_{h}\right)_{\Omega}-\left(\left\{\left\{\widehat{\boldsymbol{q}_{h}}\right\}\right\}, \llbracket \boldsymbol{v}_{h} \rrbracket_{T}\right)_{\mathcal{F}}+ \\
&\left(\llbracket \widehat{\boldsymbol{q}_{h}} \rrbracket_{T},\left\{\left\{\boldsymbol{v}_{h}\right\}\right\}\right)_{\mathcal{F} o}=\left(\boldsymbol{f}_{h}, \boldsymbol{v}_{h}\right)_{\Omega}, \forall \boldsymbol{v}_{h} \in X_{h}^{e}, \\
&\left(\mu \boldsymbol{q}_{h}, \boldsymbol{w}_{h}\right)_{\Omega}=\left(\nabla_{h} \times \boldsymbol{E}_{h}, \boldsymbol{w}_{h}\right)_{\Omega}-\left(\left\{\left\{\widehat{\boldsymbol{E}_{h}}-\boldsymbol{E}_{h}\right\}\right\}, \llbracket \boldsymbol{w}_{h} \rrbracket_{T}\right)_{\mathcal{F} o}+ \\
&\left(\llbracket \widehat{\boldsymbol{E}_{h}}-\boldsymbol{E}_{h} \rrbracket_{T},\left\{\left\{\boldsymbol{w}_{h}\right\}\right\}_{T}\right)_{\mathcal{F}}, \forall \boldsymbol{w}_{h} \in X_{h}^{e},
\end{aligned}
$$


where we have first integrated by parts in the second equation in (3.1) and used the identity

$$
\sum_{k}\left(\boldsymbol{n} \times \boldsymbol{u}_{h}, \boldsymbol{v}_{h}\right)_{\partial \mathrm{D}^{k}}=\left(\llbracket \boldsymbol{u}_{h} \rrbracket_{T},\left\{\left\{\boldsymbol{v}_{h}\right\}\right\}\right)_{\mathcal{F}}-\left(\left\{\left\{\boldsymbol{u}_{h}\right\}\right\}, \llbracket \boldsymbol{v}_{h} \rrbracket_{T}\right)_{\mathcal{F}^{o}}
$$

Among several possibilities for the numerical fluxes, we use

$$
\widehat{\boldsymbol{q}_{h}}=\left\{\left\{\boldsymbol{q}_{h}\right\}\right\}-\tau \llbracket \boldsymbol{E}_{h} \rrbracket_{T}, \text { on } \mathcal{F}, \quad \widehat{\boldsymbol{E}_{h}}= \begin{cases}\left\{\left\{\boldsymbol{E}_{h}\right\}\right\} & \text { on } \mathcal{F}^{o}, \\ 0 & \text { on } \mathcal{F}^{\partial},\end{cases}
$$

where we have assumed a perfectly-electrically-conducting (PEC) boundary condition on $\partial \Omega$. Inserting these expressions into (3.3), we obtain

$$
\begin{aligned}
\left(\boldsymbol{q}_{h}, \nabla_{h} \times \boldsymbol{v}_{h}\right)_{\Omega}-\omega^{2}\left(\varepsilon \boldsymbol{E}_{h}, \boldsymbol{v}_{h}\right)_{\Omega}-\left(\left\{\left\{\boldsymbol{q}_{h}\right\}\right\}, \llbracket \boldsymbol{v}_{h} \rrbracket_{T}\right)_{\mathcal{F}}+ \\
\left(\tau \llbracket \boldsymbol{E}_{h} \rrbracket_{T}, \llbracket \boldsymbol{v}_{h} \rrbracket_{T}\right)_{\mathcal{F}}=\left(\boldsymbol{f}_{h}, \boldsymbol{v}_{h}\right)_{\mathrm{D}^{k}}, \forall \boldsymbol{v}_{h} \in X_{h}^{e}, \\
\left(\mu \boldsymbol{q}_{h}, \boldsymbol{w}_{h}\right)_{\Omega}=\left(\nabla_{h} \times \boldsymbol{E}_{h}, \boldsymbol{w}_{h}\right)_{\Omega}-\left(\llbracket \boldsymbol{E}_{h} \rrbracket_{T},\left\{\left\{\boldsymbol{w}_{h}\right\}\right\}_{T}\right)_{\mathcal{F}}, \forall \boldsymbol{w}_{h} \in X_{h}^{e} .
\end{aligned}
$$

We observe in the second equation of (3.6), that the additional unknown $\boldsymbol{q}_{h}$ can be computed locally and removed from the equations; i.e., expressing the problem as a system is done merely for notation convenience. Indeed, introducing the lift operator (see [2] for a similar development)

$$
\begin{aligned}
r:\left(L^{2}(\mathcal{F})\right)^{3} & \longrightarrow X_{h} ; \\
\varphi & \mapsto r(\varphi) \text { such that }(r(\varphi), \eta)_{\Omega}=(\varphi,\{\{\eta\}\})_{\mathcal{F}}, \quad \forall \eta \in X_{h},
\end{aligned}
$$

and assuming that $\mu$ is piecewise constant on each element, we have

$$
\boldsymbol{q}_{h}=\mu^{-1} \nabla_{h} \times \boldsymbol{E}_{h}-\mu^{-1} r\left(\llbracket \boldsymbol{E}_{h} \rrbracket_{T}\right) .
$$

Inserting this last equation into the first equation of (3.6) and using the lift operator $r(\cdot)$, we recover

$$
\begin{aligned}
a_{h}\left(\boldsymbol{u}_{h}, \boldsymbol{v}_{h}\right):= & \left(\mu^{-1} \nabla_{h} \times \boldsymbol{u}_{h}, \nabla_{h} \times \boldsymbol{v}_{h}\right)_{\Omega}-\omega^{2}\left(\varepsilon \boldsymbol{u}_{h}, \boldsymbol{v}_{h}\right)_{\Omega}- \\
& \left(\llbracket \boldsymbol{u}_{h} \rrbracket_{T},\left\{\left\{\mu^{-1} \nabla_{h} \times \boldsymbol{v}_{h}\right\}\right\}\right)_{\mathcal{F}}-\left(\left\{\left\{\mu^{-1} \nabla_{h} \times \boldsymbol{u}_{h}\right\}\right\}, \llbracket \boldsymbol{v}_{h} \rrbracket_{T}\right)_{\mathcal{F}}+ \\
& \left(\mu^{-1} r\left(\llbracket \boldsymbol{u}_{h} \rrbracket_{T}\right), r\left(\llbracket \boldsymbol{v}_{h} \rrbracket_{T}\right)\right)_{\Omega}+\left(\tau \llbracket \boldsymbol{u}_{h} \rrbracket_{T}, \llbracket \boldsymbol{v}_{h} \rrbracket_{T}\right)_{\mathcal{F}}, \\
f_{h}\left(\boldsymbol{v}_{h}\right):= & \left(\boldsymbol{f}_{h}, \boldsymbol{v}_{h}\right)_{\Omega} .
\end{aligned}
$$

Note that the system is symmetric for real valued materials. The parameter $\tau$ is introduced to control the large null-space and is generally taken to be $\tau \propto p^{2} / h$, with $h$ being a measure of the local grid spacing and $p$ the order of the local approximation. See [13] for more details and further references for this scheme. The extension to the adjoint problem is straightforward.

Finally we emphasize that we can use different meshes for the primal and the dual problems to respect their specific natures. As a result, the approximation spaces (denoted by $X_{h}^{p}$ and $X_{h}^{d}$ ) are not the same. Neither are the bilinear forms, denoted by $a_{h}^{p}(\cdot, \cdot)$ and $a_{h}^{d}(\cdot, \cdot)$. 
4. The certified reduced basis method. In the following we discuss in some detail the construction and analysis of the certified reduced basis method, combined with the discontinuous Galerkin approximation, of the primal (1.2) and dual problem (1.3). The reliability of the formulation is secured via the construction of a posteriori error estimators.

4.1. Some notation and basic assumptions. Let $X_{h}^{p}$ (resp. $X_{h}^{d}$ ) be a discontinuous Galerkin approximation space well adapted to the primal problem (1.2) (resp. to the dual problem (1.3)) and let $X_{h}^{p d}$ be a third approximation space satisfying $X_{h}^{p} \subset X_{h}^{p d}, X_{h}^{d} \subset X_{h}^{p d}$. Associated with these spaces we introduce the corresponding discrete norms and linear and bilinear forms

$$
\begin{aligned}
\|\cdot\|_{X_{h}^{m}}: & X_{h}^{m} \longrightarrow \mathbb{R}^{+}, \\
f_{h}^{m}(\cdot ; \nu): & X_{h}^{m} \longrightarrow \mathbb{C}, \quad l_{h}^{m}(\cdot ; \nu): X_{h}^{m} \longrightarrow \mathbb{C}, \\
a_{h}^{m}(\cdot, \cdot ; \nu): & X_{h}^{m} \times X_{h}^{m} \longrightarrow \mathbb{C},
\end{aligned}
$$

with $m \in\{p, d, p d\}$. We assume throughout that the approximations $f_{h}^{m}(\cdot ; \nu), l_{h}^{m}(\cdot ; \nu)$ are linear operators that are continuous for any $\nu \in \mathcal{D}$ and that $a_{h}^{m}(\cdot, \cdot ; \nu)$ are bilinear operators continuous for any $\nu \in \mathcal{D}$; that is,

$$
\gamma^{m}(\nu):=\sup _{v_{h} \in X_{h}^{m}} \sup _{w_{h} \in X_{h}^{m}} \frac{\left|a_{h}^{m}\left(v_{h}, w_{h} ; \nu\right)\right|}{\|v\|_{X_{h}^{m}}\|w\|_{X_{h}^{m}}}<+\infty, \quad \forall \nu \in \mathcal{D} .
$$

We furthermore assume that the discrete inf-sup parameters, defined as

$$
\mid \begin{array}{ll}
\beta^{p, m}(\nu):=\inf _{v_{h} \in X_{h}^{m}} \sup _{w_{h} \in X_{h}^{m}} \frac{\left|a_{h}^{m}\left(v_{h}, w_{h} ; \nu\right)\right|}{\left\|v_{h}\right\|_{X_{h}^{m}}\left\|w_{h}\right\|_{X_{h}^{m}}}, \quad m \in\{p, p d\}, \\
\beta^{d, m}(\nu):=\inf _{\psi_{h} \in X_{h}^{m}} \sup _{\phi_{h} \in X_{h}^{m}} \frac{\left|a_{h}^{m}\left(\phi_{h}, \psi_{h} ; \nu\right)\right|}{\left\|\phi_{h}\right\|_{X_{h}^{m}}\left\|\psi_{h}\right\|_{X_{h}^{m}}}, \quad m \in\{d, p d\},
\end{array}
$$

are bounded away from zero

$$
\mid \begin{aligned}
& 0<\beta_{0}^{p, m} \leq \beta^{p, m}(\nu), \quad \nu \in \mathcal{D}, \quad \forall m \in\{p, p d\}, \\
& 0<\beta_{0}^{d, m} \leq \beta^{d, m}(\nu), \quad \nu \in \mathcal{D}, \quad \forall m \in\{d, p d\} .
\end{aligned}
$$

This suffices to guarantee existence and uniqueness of the solution to the discrete problem. It is worth noting that for Maxwell's equations, this assumption is violated for certain parameters corresponding to pure resonances. As a result, the problem is not well-defined, let alone the applicability of the reduced basis method. Fortunately, as we will show later, one can obtain an efficient lower bound of the inf-sup number inexpensively and thus identify parameters that violate this inf-sup stability condition. In fact, all the discussions in this paper should be understood with these parameters excluded.

It will furthermore be assumed that for $m \in\{p, d\}$ we have

$$
\begin{aligned}
\left\|u_{h}\right\|_{X_{h}^{m}} & =\left\|u_{h}\right\|_{X_{h}^{p d}}, & & \forall u_{h} \in X_{h}^{m}, \\
a_{h}^{m}\left(u_{h}, v_{h} ; \nu\right) & =a_{h}^{p d}\left(u_{h}, v_{h} ; \nu\right), & & \forall\left(u_{h}, v_{h}\right) \in X_{h}^{m} \times X_{h}^{m}, \\
f_{h}^{m}\left(u_{h} ; \nu\right) & =f_{h}^{p d}\left(u_{h} ; \nu\right), & & \forall u_{h} \in X_{h}^{m}, \\
l_{h}^{m}\left(u_{h} ; \nu\right) & =l_{h}^{p d}\left(u_{h} ; \nu\right), & & \forall u_{h} \in X_{h}^{m} .
\end{aligned}
$$

Denote $\mathcal{N}^{m}=\operatorname{dim}\left(X_{h}^{m}\right), m \in\{p, d, p d\}$; that is, $\mathcal{N}^{m}$ represents the total number of degrees of freedom for the discrete approximation. 
4.1.1. The truth approximation. The approximate primal and dual problems are defined as

$$
\begin{aligned}
& \mid \begin{array}{l}
\text { Find } u_{h}^{m}(\nu) \in X_{h}^{m} \text { such that }(m \in\{p, p d\}) \\
a_{h}^{m}\left(u_{h}^{m}(\nu), v_{h} ; \nu\right)=f_{h}^{m}\left(v_{h} ; \nu\right), \quad \forall v_{h} \in X_{h}^{m},
\end{array} \\
& \mid \begin{array}{l}
\text { Find } \psi_{h}^{m}(\nu) \in X_{h}^{m} \text { such that }(m \in\{d, p d\}) \\
a_{h}^{m}\left(\phi_{h}, \psi_{h}^{m}(\nu) ; \nu\right)=l_{h}^{m}\left(\phi_{h} ; \nu\right), \quad \forall \phi_{h} \in X_{h}^{m} .
\end{array}
\end{aligned}
$$

We call $u_{h}^{m}(\nu)\left(\right.$ resp. $\left.\psi_{h}^{m}(\nu)\right)$ the primal (resp. dual) truth approximation computed on $X_{h}^{m}, m \in\{p, p d\}$ (resp. $m \in\{d, p d\}$ ).

The truth approximation of the output of interest is computed as

$$
s_{h}^{p d}(\nu):=l_{h}^{p d}\left(u_{h}^{p d}(\nu) ; \nu\right)=f_{h}^{p d}\left(\psi_{h}^{p d}(\nu) ; \nu\right) .
$$

The approximation spaces are assumed to have been chosen such that $\forall m \in\{p, p d\}$, $\forall n \in\{d, p d\}$

$$
\left\|u_{h}^{m}-u^{e}\right\| \leq \varepsilon, \quad\left\|\psi_{h}^{n}-\psi^{e}\right\| \leq \varepsilon, \quad \forall \nu \in \mathcal{D} .
$$

This implies in general that $\mathcal{N}^{m}$ will have to be large, resulting in a significant computational cost for problems where many instances of $\nu$ must be evaluated. One of the goals of this work is to significantly reduce this cost.

We conclude this subsection by a remark on $X_{h}^{p d}, u_{h}^{p d}$ and $\psi_{h}^{p d}$. Different primal and dual spaces, $X_{h}^{p}$ and $X_{h}^{d}$ are introduced to respect the different natures of the primal and dual solutions. A third (fine) space is introduced to include the two former spaces, a necessary step for analysis. In practice, we do not solve the (larger) problem in $X_{h}^{p d}$.

4.2. The reduced basis method. The primary goal here is to reduce the dimension of the approximation spaces to speed up the computations without impacting the accuracy. To facilitate this we introduce two sets of samples

$$
\mid \begin{aligned}
& \mathcal{S}_{N}^{p}=\left\{\nu_{i}^{p} \in \mathcal{D}, 1 \leq i \leq N\right\}, \\
& \mathcal{S}_{M}^{d}=\left\{\nu_{j}^{d} \in \mathcal{D}, 1 \leq j \leq M\right\},
\end{aligned}
$$

and the associated reduced basis spaces

$$
\mid \begin{aligned}
& X_{N}^{p}=\operatorname{span}\left\{u_{h}^{p}\left(\nu_{i}^{p}\right), 1 \leq i \leq N\right\}, \\
& X_{M}^{d}=\operatorname{span}\left\{\psi_{h}^{d}\left(\nu_{j}^{d}\right), 1 \leq j \leq M\right\} .
\end{aligned}
$$

Solving the primal and dual reduced basis problems

$$
\begin{aligned}
& \mid \begin{array}{l}
\text { Find } u_{N}(\nu) \in X_{N}^{p} \text { such that } \\
a_{h}^{p}\left(u_{N}(\nu), v_{N} ; \nu\right)=f_{h}^{p}\left(v_{N} ; \nu\right), \quad \forall v_{N} \in X_{N}^{p},
\end{array} \\
& \mid \begin{array}{l}
\text { Find } \psi_{M}(\nu) \in X_{M}^{d} \text { such that } \\
a_{h}^{d}\left(\phi_{M}, \psi_{M}(\nu) ; \nu\right)=l_{h}^{d}\left(\phi_{M} ; \nu\right), \quad \forall \phi_{M} \in X_{M}^{d},
\end{array}
\end{aligned}
$$

we define the reduced basis approximation of the output as [29]

$$
s_{N, M}(\nu)=l^{p}\left(u_{N}(\nu) ; \nu\right)-a^{p d}\left(u_{N}(\nu), \psi_{M}(\nu) ; \nu\right)+f^{d}\left(\psi_{M}(\nu) ; \nu\right) .
$$

We will observe numerically that when the sets of samples, Eq. (4.9), are carefully chosen, this reduced basis output converges toward the truth approximations of the output at an exponential rate [23]. 
4.3. The affine assumption and off-line/on-line strategies. One might expect a reduction of the numerical cost as soon as the computation of $u_{N}(\nu)$ (resp. $\psi_{M}(\nu)$ ) involves the solution of an $N \times N$ (resp. $M \times M$ ) linear system. However, the total computational cost still depends on $\mathcal{N}^{m}, m \in\{p, d, p d\}$ as for each $\nu$, the linear systems have to be assembled and (4.13) has to be evaluated. This bottleneck can, however, be removed in many situations.

Let us assume that the operators $f_{h}^{m}(\cdot ; \cdot), l_{h}^{m}(\cdot ; \cdot)$ and $a_{h}^{m}(\cdot, \cdot ; \cdot) m \in\{p, d, p d\}$ can be expressed as

$$
\begin{aligned}
a_{h}^{m}\left(u_{h}, v_{h} ; \nu\right) & =\sum_{q=1}^{Q_{a}} \Theta_{q}^{a}(\nu) a_{q, h}^{m}\left(u_{h}, v_{h}\right), & & \forall\left(u_{h}, v_{h}\right) \in X_{h}^{m} \times X_{h}^{m}, \\
f_{h}^{m}\left(u_{h} ; \nu\right) & =\sum_{q=1}^{Q_{f}} \Theta_{q}^{f}(\nu) f_{q, h}^{m}\left(u_{h}\right), & & \forall u_{h} \in X_{h}^{m}, \\
l_{h}^{m}\left(\phi_{h} ; \nu\right) & =\sum_{q=1}^{Q_{l}} \Theta_{q}^{l}(\nu) l_{q, h}^{m}\left(\phi_{h}\right), & & \forall \phi_{h} \in X_{h}^{m},
\end{aligned}
$$

where the $a_{q, h}^{m}(\cdot, \cdot)$ (resp. $f_{q, h}^{m}(\cdot)$ and $\left.l_{q, h}^{m}(\cdot)\right)$ are $\nu$-independent discrete operators and the functions $\Theta_{q}^{s}(\cdot), s \in\{a, f, l\}$ depend only on $\nu$. We generally assume that $Q_{s}, s \in\{a, f, l\}$ is small although this is not an essential assumption.

Equation in (4.14) is referred to as the affine assumptions for $f_{h}^{m}(\cdot ; \cdot), l_{h}^{m}(\cdot ; \cdot)$ and $a_{h}^{m}(\cdot, \cdot ; \cdot) m \in\{p, d, p d\}$. As we will see shortly, this is what enables the development of an attractive off-line/on-line strategy. The off-line part of the computation, being $\nu$ independent, can be done entirely in a preprocessing stage. The computational cost of the on-line part is $\mathcal{N}^{m}$-independent and thus, very small.

To further emphasize this, we write the reduced basis solutions as linear combinations of the elements of the reduced basis:

$$
u_{N}(\nu)=\sum_{i=1}^{N} u_{N}^{i}(\nu) \xi_{i}^{p}, \quad \psi_{N}(\nu)=\sum_{j=1}^{M} \psi_{M}^{j}(\nu) \xi_{j}^{d} .
$$

Here we have introduced the basis elements, $\xi_{i}^{p}$, and $\xi_{i}^{d}$, which, in the simplest case, are $\xi_{i}^{p}=u_{h}^{p}\left(\nu_{i}\right)$ and $\xi_{i}^{d}=\psi_{h}^{d}\left(\nu_{i}\right)$. However, as we will discuss shortly, it is computationally advantageous to require the basis elements be mutually orthogonal.

In this way, solving (4.11) and (4.12) reduces to

$$
\mid \begin{aligned}
& \text { Find } u_{N}^{j}(\nu), j \in\{1, \ldots, N\} \text { such that } \\
& \sum_{j=1}^{N}\left[\sum_{q=1}^{Q_{a}} \Theta_{q}^{a}(\nu) a_{q, h}^{p}\left(\xi_{j}^{p}, \xi_{i}^{p}\right)\right] u_{N}^{j}(\nu)=\sum_{q=1}^{Q_{f}} \Theta_{q}^{f}(\nu) f_{q, h}^{p}\left(\xi_{i}^{p}\right), \quad i \in\{1, \ldots, N\},
\end{aligned}
$$

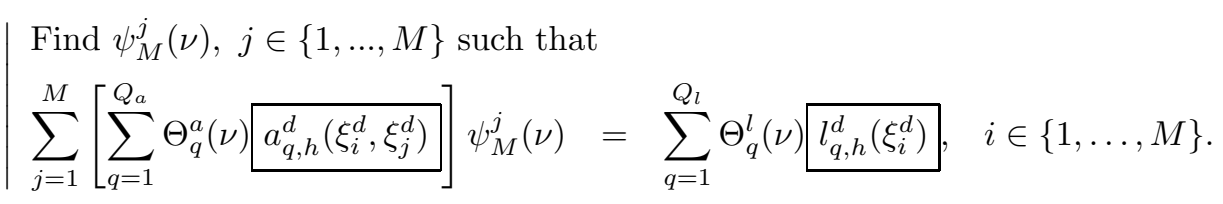

where we have invoked (4.14). The framed terms are all $\nu$-independent and can be precomputed off-line. Once these computations have been done, the number of 
operations to be performed in the on-line procedure is proportional to

$$
\begin{array}{cl}
N^{2} Q_{a}+M^{2} Q_{a}+ & \text { (assembling of the matrices) } \\
N Q_{f}+M Q_{l}+ & \text { (assembling of the right hand sides) } \\
N^{3}+M^{3}, & \text { (solving the full linear systems) }
\end{array}
$$

and it is therefore very fast as it does not depend on the dimension of the truth approximation spaces, $\mathcal{N}^{m}$.

Using the affine assumption, the reduced basis output can be expressed as

$$
\begin{aligned}
s_{N, M}(\nu)= & \sum_{i=1}^{N} \sum_{q=1}^{Q_{l}} u_{N}^{i}(\nu) \Theta_{q}^{l}(\nu) l_{q, h}^{p}\left(\xi_{i}^{p}\right)+\sum_{j=1}^{M} \sum_{q=1}^{Q_{f}} \psi_{M}^{j}(\nu) \Theta_{q}^{f}(\nu) f_{q, h}^{d}\left(\xi_{j}^{d}\right) \\
& -\sum_{i=1}^{N} \sum_{j=1}^{M} \sum_{q=1}^{Q_{a}} u_{N}^{i}(\nu) \psi_{M}^{j}(\nu) \Theta_{q}^{a}(\nu) a_{q, h}^{p d}\left(\xi_{i}^{p}, \xi_{j}^{d}\right) .
\end{aligned}
$$

The framed terms can again be computed in preprocessing as they are independent of $\nu$. The number of operations depending on $\nu$ is of order

$$
N Q_{l}+M Q_{f}+N M Q_{a}
$$

which is independent of $\mathcal{N}^{m}$.

4.4. A priori estimates. In this section we discuss the stability of problems (4.11) and (4.12) and the convergence of the reduced basis approximations toward the truth approximations for increasing $N$ and $M$.

We begin by pointing out that stability of the reduced basis problem is not implied by the assumptions on (4.3). It will be satisfied only for certain sets of samples (4.9). Let us therefore consider stability of the reduced basis problems.

TheOREm 4.1 (Stability of (4.11) and (4.12)). Assume that the discrete inf-sup parameters satisfy

$$
\mid \begin{gathered}
0<\tilde{\beta}_{0}^{p} \leq \inf _{v_{N} \in X_{N}^{p}} \sup _{w_{N} \in X_{N}^{p}} \frac{\left|a_{h}^{p}\left(v_{N}, w_{N} ; \nu\right)\right|}{\left\|v_{N}\right\|_{X_{N}^{p}}\left\|w_{N}\right\|_{X_{N}^{p}}}, \\
0<\tilde{\beta}_{0}^{d} \leq \inf _{\psi_{M} \in X_{M}^{d}} \sup _{\phi_{M} \in X_{M}^{d}} \frac{\left|a_{h}^{d}\left(\phi_{M}, \psi_{M} ; \nu\right)\right|}{\left\|\phi_{M}\right\|_{X_{M}^{d}}\left\|\psi_{M}\right\|_{X_{M}^{d}}} .
\end{gathered}
$$

$\forall \nu \in \mathcal{D}$.

Then, (4.11) and (4.12) are stable.

Proof: The existence and uniqueness of the solution of problem (4.11) (resp. (4.12)) are ensured by assumption (4.16(a)) (resp. (4.16(b)). Moreover, we deduce from the same assumption that there $\exists \chi_{a_{h}^{p}\left(u_{N}(\nu), ;, \nu\right)}^{X_{p}^{p}} \in X_{N}^{p}\left(\chi_{f(\cdot)}^{X} \in X\right.$ denotes the Riesz representation of any continuous linear form $f(\cdot)$ defined on $X$ ) such that

$$
\begin{aligned}
\tilde{\beta}_{0}^{p}\left\|u_{N}(\nu)\right\|_{X_{N}^{p}}\left\|\chi_{a_{h}^{p}\left(u_{N}(\nu), ; \nu \nu\right)}^{X_{N}^{p}}\right\|_{X_{N}^{p}} & \leq\left|a_{h}^{p}\left(u_{N}(\nu), \chi_{a_{h}^{p}\left(u_{N}(\nu), ; \nu\right)}^{X_{N}^{p}} ; \nu\right)\right| \\
& =\left|f_{h}^{p}\left(\chi_{a_{h}^{p}\left(u_{N}(\nu), ; ; \nu\right)}^{X_{p}^{p}} ; \nu\right)\right| \\
& \leq\left\|f_{h}^{p}(\cdot ; \nu)\right\|_{\left(X_{N}^{p}\right)^{\prime}}\left\|\chi_{a^{p}\left(u_{N}(\nu), ; \nu\right.}^{X_{N}^{p}}\right\|_{X_{N}^{p}}^{p} .
\end{aligned}
$$


This implies

$$
\left\|u_{N}(\nu)\right\|_{X_{h}^{p}} \leq \frac{1}{\tilde{\beta}_{0}^{p}}\left\|f_{h}^{p}(\cdot ; \nu)\right\|_{\left(X_{h}^{p}\right)^{\prime}}
$$

that is, continuity of the solution with respect to the data. A similar proof can be done for the dual problem.

TheOREM 4.2 (A priori primal and dual estimates). Under the same assumptions as Theorem 4.1 we have

$$
\begin{aligned}
\left\|u_{h}^{m}(\nu)-u_{N}(\nu)\right\|_{X_{h}^{m}} & \leq \mathcal{C}_{p, m}(\nu) \inf _{v_{N} \in X_{N}^{p}}\left\|u_{h}^{m}(\nu)-v_{N}\right\|_{X_{h}^{m}}, \quad m \in\{p, p d\}, \\
\left\|\psi_{h}^{m}(\nu)-\psi_{M}(\nu)\right\|_{X_{h}^{m}} & \leq \mathcal{C}_{d, m}(\nu) \inf _{\phi_{M} \in X_{M}^{d}}\left\|\psi_{h}^{m}(\nu)-\phi_{M}\right\|_{X_{h}^{m}}, \quad m \in\{d, p d\} .
\end{aligned}
$$

where

$$
\mathcal{C}_{n, m}(\nu)=1+\frac{\gamma^{m}(\nu)}{\tilde{\beta}_{0}^{n}}, \quad n \in\{p, d\}, m \in\{p, d, p d\}
$$

Proof: Using (4.16) we know that, for any $\varepsilon>0$ and any $v_{N} \in X_{N}^{p}$, there $\exists w_{N} \in X_{N}^{p}$ such that

$$
\begin{aligned}
& \left(\tilde{\beta}_{0}^{p}-\varepsilon\right)\left\|u_{N}(\nu)-v_{N}\right\|_{X_{N}^{p}}\left\|w_{N}\right\|_{X_{h}^{p}} \leq\left|a_{h}^{p}\left(u_{N}(\nu)-v_{N}, w_{N} ; \nu\right)\right| \\
& =\left|a_{h}^{m}\left(u_{h}^{m}(\nu)-v_{N}, w_{N} ; \nu\right)\right| \leq \gamma^{m}(\nu)\left\|u_{h}^{m}(\nu)-v_{N}\right\|_{X_{h}^{m}}\left\|w_{N}\right\|_{X_{N}^{p}} .
\end{aligned}
$$

Thus, we prove the result for the primal problem $(4.17(\mathrm{a}))$ by using the triangle inequality. The result for the dual problem $(4.17(\mathrm{~b}))$ is obtained in a similar way.

Before giving a similar result for the output we note that

$$
s_{h}^{p d}(\nu)-s_{N, M}(\nu)=a_{h}^{p d}\left(u_{h}^{p d}(\nu)-u_{N}(\nu), \psi_{h}^{p d}(\nu)-\psi_{M}(\nu) ; \nu\right),
$$

using $(4.5)-(4.7)$ and linearity.

THEOREM 4.3 (A priori estimate on the output with respect to $s_{h}^{p d}(\nu)$ ). Under assumptions as in Theorem 4.1 we have

$$
\begin{aligned}
\left|s_{h}^{p d}(\nu)-s_{N, M}(\nu)\right| \leq \gamma^{p d}(\nu) \mathcal{C}_{p, p d}(\nu) \mathcal{C}_{d, p d}(\nu) \times \\
\inf _{v_{N} \in X_{N}^{p}}\left\|u_{h}^{p d}(\nu)-v_{N}\right\|_{X_{h}^{p d}} \inf _{\phi_{M} \in X_{M}^{d}}\left\|\psi_{h}^{p d}(\nu)-\phi_{M}\right\|_{X_{h}^{p d}}, \\
\left|s_{h}^{p d}(\nu)-s_{N, M}(\nu)\right| \leq \gamma^{p d}(\nu) \times \\
{\left[\left\|u_{h}^{p d}(\nu)-u_{h}^{p}(\nu)\right\|_{X_{h}^{p d}}+\mathcal{C}_{p, p}(\nu) \inf _{v_{N} \in X_{N}^{p}}\left\|u_{h}^{p}(\nu)-v_{N}\right\|_{X_{N}^{p}}\right] \times } \\
{\left[\left\|\psi_{h}^{p d}(\nu)-\psi_{h}^{d}(\nu)\right\|_{X_{h}^{p d}}+\mathcal{C}_{d, d}(\nu) \inf _{\phi_{M} \in X_{M}^{d}}\left\|\psi_{h}^{d}(\nu)-\phi_{M}\right\|_{X_{M}^{d}}\right] . }
\end{aligned}
$$


Proof: Using (4.18) and the continuity of $a_{h}^{m}(\cdot, \cdot ; \nu)$ we obtain

$$
\left|s_{h}^{p d}(\nu)-s_{N, M}(\nu)\right| \leq \gamma^{p d}(\nu)\left\|u_{h}^{p d}(\nu)-u_{N}(\nu)\right\|_{X_{h}^{p d}}\left\|\psi_{h}^{p d}(\nu)-\psi_{M}(\nu)\right\|_{X_{h}^{p d}}
$$

Then we use inequalities (4.17) with $m=p d$ to obtain (4.19). To prove (4.20), we employ the triangle inequality on the last expression and apply the first (resp. second) inequality on (4.17) with $m=p$ (resp. $m=d$ ).

4.5. A posteriori estimates. We are now in a position to provide a posteriori error estimators that certify the reduced basis approximation with respect to the truth approximation. The evaluation of these estimators will be done following an off-line/on-line strategy similar to the one discussed in Section 4.3.

We start by introducing some helpful notation. For each $w_{N} \in X_{N}^{p}\left(\right.$ resp. $\left.\psi_{M} \in X_{M}^{d}\right)$, we define the residual for the primal (resp. dual) problem as

$$
\mid \begin{aligned}
& R_{h}^{p}\left(v_{h}, w_{N} ; \nu\right)=f_{h}^{p d}\left(v_{h} ; \nu\right)-a_{h}^{p d}\left(w_{N}, v_{h} ; \nu\right), \quad \forall v_{h} \in X_{h}^{p d}, \\
& R_{h}^{d}\left(\phi_{h}, \psi_{M} ; \nu\right)=l_{h}^{p d}\left(\phi_{h} ; \nu\right)-a_{h}^{p d}\left(\phi_{h}, \psi_{M} ; \nu\right), \quad \forall \phi_{h} \in X_{h}^{p d} .
\end{aligned}
$$

Let us also introduce their dual norms on the spaces $\left(X_{h}^{m}\right)^{\prime}, m \in\{p, d, p d\}$ as

$$
\mid \begin{aligned}
& \varepsilon_{N}^{p, m}(\nu):=\left\|R_{h}^{p}\left(\cdot, u_{N}(\nu) ; \nu\right)\right\|_{\left(X_{h}^{m}\right)^{\prime}}=\sup _{v_{h} \in X_{h}^{m}} \frac{\left|R_{h}^{p}\left(v_{h}, u_{N}(\nu) ; \nu\right)\right|}{\left\|v_{h}\right\|_{X_{h}^{m}}}, \\
& \varepsilon_{M}^{d, m}(\nu):=\left\|R_{h}^{d}\left(\cdot, \psi_{M}(\nu) ; \nu\right)\right\|_{\left(X_{h}^{m}\right)^{\prime}}=\sup _{\phi_{h} \in X_{h}^{m}} \frac{\left|R_{h}^{d}\left(\phi_{h}, \psi_{M}(\nu) ; \nu\right)\right|}{\left\|\phi_{h}\right\|_{X_{h}^{m}}} .
\end{aligned} .
$$

Note that $\varepsilon_{N}^{p, m}(\nu)$ also depends on $u_{N}$ and $\varepsilon_{M}^{d, m}(\nu)$ on $\psi_{M}$ but we have left out this explicit relationship to simplify the notation.

Assume that we can construct a lower bound for the inf-sup parameters (4.3) denoted by $\bar{\beta}^{p, m}(\nu), m \in\{p, p d\}$ and $\bar{\beta}^{d, m}(\nu), m \in\{d, p d\}$ such that

$$
\mid \begin{aligned}
& 0<\bar{\beta}_{0}^{p, m} \leq \bar{\beta}^{p, m}(\nu) \leq \beta^{p, m}(\nu), \quad \forall \nu \in \mathcal{D}, \quad \forall m \in\{p, p d\}, \\
& 0<\bar{\beta}_{0}^{d, m} \leq \bar{\beta}^{d, m}(\nu) \leq \beta^{d, m}(\nu), \quad \forall \nu \in \mathcal{D}, \quad \forall m \in\{d, p d\} .
\end{aligned}
$$

These lower bounds are assumed to be computable at a low on-line computational cost independent of the dimension of the truth approximation spaces. We explain in Sec. 5.3 one technique to effectively compute this quantity, but for now we simply assume that it is available.

4.5.1. A posteriori estimators for the reduced basis solutions. Let us define the a posteriori error estimators for the solution of the primal and dual reduced basis problems as follows:

Definition 4.4 (Primal and dual estimators). The primal and dual estimators are defined by

$$
\mid \begin{aligned}
\Delta_{N}^{p, m}(\nu) & :=\frac{\varepsilon_{N}^{p, m}(\nu)}{\bar{\beta}^{p, m}(\nu)}, \quad m \in\{p, p d\} \\
\Delta_{M}^{d, m}(\nu) & :=\frac{\varepsilon_{M}^{d, m}(\nu)}{\bar{\beta}^{d, m}(\nu)}, \quad m \in\{d, p d\}
\end{aligned}
$$


We have the following theorem concerning their efficiency

TheOREM 4.5. The efficiency of the primal estimators, $m \in\{p, p d\}$

$$
\eta_{N}^{p, m}(\nu):=\frac{\Delta_{N}^{p, m}(\nu)}{\left\|u_{N}(\nu)-u_{h}^{m}(\nu)\right\|_{X_{h}^{m}}}
$$

satisfies, $m \in\{p, p d\}$

$$
1 \leq \eta_{N}^{p, m}(\nu) \leq \frac{\gamma^{m}(\nu)}{\bar{\beta}^{p, m}(\nu)} ; \quad \forall \nu \in \mathcal{D}
$$

We have a similar result for the dual estimator.

Proof: This result is a consequence of the following set of inequalities

$$
\begin{aligned}
\bar{\beta}^{p, m}(\nu) \leq \inf _{v_{h} \in X_{h}^{m}} \sup _{w_{h} \in X_{h}^{m}} \frac{\left|a_{h}^{m}\left(v_{h}, w_{h} ; \nu\right)\right|}{\left\|v_{h}\right\|_{X_{h}^{m}}\left\|w_{h}\right\|_{X_{h}^{m}}}=\beta^{p, m}(\nu) \\
\leq \sup _{w_{h} \in X_{h}^{m}} \frac{\left|a_{h}^{m}\left(u_{h}^{m}(\nu)-u_{N}(\nu), w_{h} ; \nu\right)\right|}{\left\|u_{h}^{m}(\nu)-u_{N}(\nu)\right\|_{X_{h}^{m}\left\|w_{h}\right\|_{X_{h}^{m}}}}=\eta_{N}^{p, m}(\nu) \bar{\beta}^{p, m}(\nu) \\
\leq \sup _{v_{h} \in X_{h}^{m}} \sup _{w_{h} \in X_{h}^{m}} \frac{\left|a_{h}^{m}\left(v_{h}, w_{h} ; \nu\right)\right|}{\left\|v_{h}\right\|_{X_{h}^{m}}\left\|w_{h}\right\|_{X_{h}^{m}}}=\gamma^{m}(\nu) .
\end{aligned}
$$

Dividing by $\bar{\beta}^{p, m}(\nu)$ we obtain (4.25).

4.5.2. A posteriori estimator for the reduced basis output. Recall that our goal is to estimate the output $s(u)$ rather than the solution of the problem. Using (4.18) we obtain

$$
\begin{aligned}
s_{h}^{p d}(\nu)-s_{N, M}(\nu)= & R_{h}^{d}\left(u_{h}^{p d}(\nu)-u_{N}(\nu), \psi_{M}(\nu) ; \nu\right) \\
= & R_{h}^{d}\left(u_{h}^{p}(\nu)-u_{N}(\nu), \psi_{M}(\nu) ; \nu\right)+ \\
& a_{h}^{p d}\left(u_{h}^{p d}(\nu)-u_{h}^{p}(\nu), \psi_{h}^{p d}(\nu)-\psi_{M}(\nu) ; \nu\right),
\end{aligned}
$$

which is useful for constructing the estimators. We have the following result

THEOREM 4.6 (Estimator for $s_{N, M}(\nu)$ with respect to $s_{h}^{p d}(\nu)$ ). The following inequalities are satisfied

$$
\begin{aligned}
\left|s_{h}^{p d}-s_{N, M}\right| \leq & \frac{\varepsilon_{N}^{p, p d}(\nu) \varepsilon_{M}^{d, p d}(\nu)}{\bar{\beta}^{p, p d}(\nu)}, \\
\left|s_{h}^{p d}-s_{N, M}\right| \leq & \frac{\varepsilon_{N}^{p, p}(\nu) \varepsilon_{M}^{d, p}(\nu)}{\bar{\beta}^{p, p}(\nu)}+\varepsilon_{M}^{d, p d}(\nu)\left\|u_{h}^{p d}(\nu)-u_{h}^{p}(\nu)\right\|_{X_{h}^{p d}}, \\
\left|s_{h}^{p d}-s_{N, M}\right| \leq & \frac{\varepsilon_{N}^{p, p}(\nu) \varepsilon_{M}^{d, p}(\nu)}{\bar{\beta}^{p, p}(\nu)}+\gamma^{p d}(\nu)\left\|u_{h}^{p d}(\nu)-u_{h}^{p}(\nu)\right\|_{X_{h}^{p d}} \times \\
& {\left[\frac{\varepsilon_{M}^{d, d}(\nu)}{\bar{\beta}^{d, d}(\nu)}+\left\|\psi_{h}^{p d}(\nu)-\psi_{h}^{d}(\nu)\right\|_{X_{h}^{p d}}\right] . }
\end{aligned}
$$


Proof: We use (4.27) to write

$$
\begin{aligned}
\left|s_{h}^{p d}(\nu)-s_{N, M}(\nu)\right| & =\left|R_{h}^{d}\left(u_{h}^{p d}(\nu)-u_{N}(\nu), \psi_{M}(\nu) ; \nu\right)\right| \\
& \leq \varepsilon_{M}^{d, p d}(\nu)\left\|u_{h}^{p d}(\nu)-u_{N}(\nu)\right\|_{X_{h}^{p d}}
\end{aligned}
$$

We obtain (4.29) using (4.25) with $m=p d$.

To recover (4.30) we use (4.28) to obtain

$$
\begin{aligned}
\left|s_{h}^{p d}(\nu)-s_{N, M}(\nu)\right| \leq & \left|R_{h}^{d}\left(u_{h}^{p}(\nu)-u_{N}(\nu), \psi_{M}(\nu) ; \nu\right)\right|+ \\
& \left|R_{h}^{d}\left(u_{h}^{p d}(\nu)-u_{h}^{p}(\nu), \psi_{M}(\nu) ; \nu\right)\right| \\
\leq & \varepsilon_{M}^{d, p}(\nu)\left\|u_{h}^{p}(\nu)-u_{N}(\nu)\right\|_{X_{h}^{p d}}+ \\
& \varepsilon_{M}^{d, p d}(\nu)\left\|u_{h}^{p d}(\nu)-u_{h}^{p}(\nu)\right\|_{X_{h}^{p d}} .
\end{aligned}
$$

We conclude with the same argument as above with $m=p$.

To prove (4.31), combine (4.28) with the continuity of the operator $a_{h}^{p d}(\cdot, \cdot ; \nu)$

$$
\begin{aligned}
\left|s_{h}^{p d}(\nu)-s_{N, M}(\nu)\right| \leq & \varepsilon_{M}^{d, p}(\nu)\left\|u_{h}^{p}(\nu)-u_{N}(\nu)\right\|_{X_{h}^{p d}}+ \\
& \gamma^{p d}(\nu)\left\|u_{h}^{p d}(\nu)-u_{h}^{p}(\nu)\right\|_{X_{h}^{p d}}\left\|\psi_{h}^{p d}(\nu)-\psi_{M}(\nu)\right\|_{X_{h}^{p d}},
\end{aligned}
$$

and employ the triangle inequality and Theorem 4.5 to obtain

$$
\left\|\psi_{h}^{p d}(\nu)-\psi_{M}(\nu)\right\|_{X_{h}^{p d}} \leq \frac{\varepsilon_{M}^{d, d}(\nu)}{\bar{\beta}^{d, d}(\nu)}+\left\|\psi_{h}^{p d}(\nu)-\psi_{h}^{d}(\nu)\right\|_{X_{h}^{p d}}
$$

The quantity on the right hand side of (4.29) can be used as a rigorous upper bound of the actual error on the output and can be computed using an off-line/on-line strategy. However, the off-line part of the computations involves the solution of linear systems and eigenvalue problems based on the larger space $X_{h}^{p d}$, and may, thus, become unnecessarily expensive.

On the right hand side of (4.30) and (4.31) there are some terms that are not easily computable (for example $\gamma^{p d}(\nu)$ ). For the other terms, we can use a crude estimation

$$
\left\|u_{h}^{p d}(\nu)-u_{h}^{p}(\nu)\right\|_{X_{h}^{p d}} \leq 2 \varepsilon, \quad\left\|\psi_{h}^{p d}(\nu)-\psi_{h}^{d}(\nu)\right\|_{X_{h}^{p d}} \leq 2 \varepsilon,
$$

to provide a rigorous upper bound of the error. Here $\varepsilon$ estimates the approximation error associated with the truth approximation. However, both quantities are in practice very small and it is reasonable to use

$$
\Delta_{N, M}^{s}:=\frac{\varepsilon_{N}^{p, p}(\nu) \varepsilon_{M}^{d, p}(\nu)}{\bar{\beta}^{p, p}(\nu)},
$$

as an estimator of the error. In this case, the off-line part of the algorithm involves only the solution of linear systems and eigenvalue problems based on $X_{h}^{p}$ which is likely more affordable compared to an approach based on $X_{h}^{p d}$. 
5. Computational Aspects. In the discussions so far, we have laid out the analysis of the reduced basis method but we have paid limited attention to the implementation of the method. In the following we address this central issue in more detail.

5.1. Construction of the reduced basis. An essential point in the construction of the reduced basis spaces is the selection of the sets of samples (4.9). All well-posedness and convergence properties depend on this choice. Below, for completeness, we present the algorithm for the construction of the primal and the dual reduced basis sets that generally provide good results, see also [35] and the references therein. The method is based on a greedy approach in which we build a mesh $\mathcal{S}$ of the set of parameters $\mathcal{D}$, and recursively choose the parameters $\nu_{j}^{m} \in \mathcal{S}, m \in\{p, d\}$ such that a distance between the reduced basis approximation and the truth approximation is minimized. The two reduced basis spaces $X_{N}^{p}$ and $X_{M}^{d}$ are built separately with both constructions being similar. We present the one for the primal problem:

- Choose a $q$-dimensional mesh $\mathcal{S}$ of the set of parameters $\mathcal{D}$.

- Choose the first parameter $\nu_{1}^{p}$ among the elements of the mesh $\mathcal{S}$ (randomly for example).

- Compute $u_{h}^{p}\left(\nu_{1}^{p}\right)$.

- Initialize the reduced basis space $X_{1}^{p}=\operatorname{span}\left\{u_{h}^{p}\left(\nu_{1}^{p}\right)\right\}$.

- For $j=2, \ldots, N$

- Choose the next sample as

$$
\nu_{j}^{p}:=\arg \max _{\nu \in \mathcal{S}} \Delta_{j-1}^{p, p}(\nu) .
$$

where $\Delta_{j-1}^{p, p}(\nu)$ is defined in Def. 4.4 .

- Compute $u_{h}^{p}\left(\nu_{j}^{p}\right)$.

- Update the reduced basis space: $X_{j}^{p}=\operatorname{span}\left\{u_{h}^{p}\left(\nu_{i}^{p}\right), i \in\{1, \ldots, j\}\right\}$. The dimension of the updated reduced basis is $j$.

Let us make a few remarks:

REMARK 5.1. In criteria (5.1), we seek to add those values of the parameters, $\nu_{j}^{p}$, for which the error between $u_{h}^{p}(\nu)$ and $u_{N}(\nu)$ is maximized. This means choosing

$$
\widetilde{\nu_{j}^{p}}:=\arg \max _{\nu \in \mathcal{S}}\left\|u_{h}^{p}(\nu)-u_{N}(\nu)\right\|_{X_{h}^{p}}
$$

To accomplish this it appears we would need to compute the primal truth approximation for all $\nu \in \mathcal{S}$, leading to an expensive procedure. However, as soon as the estimator $\Delta_{j-1}^{p, p}(\nu)$ is accurate, it enables a rigorous upper bound of the actual error with a low evaluation cost and we use it as

$$
\left\|u_{h}^{p}(\nu)-u_{N}(\nu)\right\|_{X_{h}^{p}} \leq \Delta_{j-1}^{p, p}(\nu) .
$$

In this way, the primal truth approximation is just computed for the $N$ selected parameters. As a consequence, the numerical method is much cheaper while, as we have numerically observed, the basis quality is comparable.

REMARK 5.2. It is important to choose a suitable basis for the representation of the space $X_{N}^{p}$ to avoid stability issues. If we use the native basis given as $\left\{u_{h}^{p}\left(\nu_{1}^{p}\right), \ldots, u_{h}^{p}\left(\nu_{N}^{p}\right)\right\}$ the condition number of the matrix associated with problem (4.11) grows exponentially with $N$. However, this is easily overcome by using an orthogonalization process such as the Gram-Schmidt method. After this, the condition number of the reduced basis 
problem inherits the properties of the matrix associated with the truth approximation (4.5). For the discussion on the inf-sup condition of the final reduced system (4.11), the readers are referred to [33].

REMARK 5.3. Note that we can build the two basis sets simultaneously by directly minimizing the error on the output rather than the errors in the primal and dual solutions. We could therefore expect a reduction in the dimension of the reduced basis spaces in order to obtain a prescribed accuracy on the output. This method is outlined as follows:

- Choose a q-dimensional mesh $\mathcal{S}$ of the set of parameters $\mathcal{D}$.

- Choose $\nu_{1}^{p}=\nu_{1}^{d}$ among the elements of the mesh $\mathcal{S}$ (randomly for example).

- Compute $u_{h}^{p}\left(\nu_{1}^{p}\right)$ and $\psi_{h}^{d}\left(\nu_{1}^{d}\right)$.

- Initialize the reduced basis spaces

$$
X_{1}^{p}=\operatorname{span}\left\{u_{h}^{p}\left(\nu_{1}^{p}\right)\right\} \quad \text { and } X_{1}^{d}=\operatorname{span}\left\{\psi_{h}^{d}\left(\nu_{1}^{d}\right)\right\} .
$$

- For $j=2, \ldots, N$

- Choose the next sample as

$$
\nu_{j}^{p}=\nu_{j}^{d}:=\arg \max _{\nu \in \mathcal{S}} \Delta_{j-1, j-1}^{s}(\nu) .
$$

where $\Delta_{j-1, j-1}^{s}(\nu)$ is defined by (4.32).

- Compute $u_{h}^{p}\left(\nu_{j}^{p}\right)$ and $\psi_{h}^{d}\left(\nu_{j}^{d}\right)$.

- Update the reduced basis spaces:

$$
\begin{aligned}
& X_{j}^{p}=\operatorname{span}\left\{u_{h}^{p}\left(\nu_{i}^{p}\right), i \in\{1, \ldots, j\}\right\}, \\
& X_{j}^{d}=\operatorname{span}\left\{\psi_{h}^{d}\left(\nu_{i}^{d}\right), i \in\{1, \ldots, j\}\right\} .
\end{aligned}
$$

The dimension of the updated reduced basis is $j$.

Although our current numerical experiments indicate no major benefits of using this output-oriented algorithm, we believe it is essential and should make a difference in cases such as when the primal/dual solutions have oscillations and the output functional involves averaging.

5.2. Computing $\varepsilon_{N}^{p, m}(\nu), m \in\{p, d, p d\}$. In this section we discuss how to compute $\varepsilon_{N}^{p, m}$ following an off-line/on-line strategy. Recall that these quantities, introduced in (4.22), are nothing but the dual norms of the primal residual on the space $X_{h}^{m}$. Using the Riesz theorem we know that

$$
\exists \chi_{R_{h}^{p}\left(\cdot, u_{N}(\nu) ; \nu\right)}^{X_{m}^{m}} \in X_{h}^{m} \text { such that } \varepsilon_{N}^{p, m}=\left\|\chi_{R_{h}^{p}\left(\cdot, u_{N}(\nu) ; \nu\right)}^{X_{m}^{m}}\right\|_{X_{h}^{m}},
$$

where $\chi_{f(\cdot)}^{X} \in X$ denotes the Riesz representation of any continuous linear form $f(\cdot)$ defined on $X$. Moreover, $\chi_{R_{h}^{p}\left(\cdot, u_{N}(\nu) ; \nu\right)}^{X_{m}^{m}}$ is characterized by

$$
\left(\chi_{R_{h}^{p}\left(\cdot, u_{N}(\nu) ; \nu\right)}^{X_{m}^{m}}, v_{N}\right)_{X_{h}^{m}}=R_{h}^{p}\left(v_{N}, u_{N}(\nu) ; \nu\right), \quad \forall v_{N} \in X_{h}^{m} .
$$

Inserting (4.15) into (4.21) and using the affine assumption (4.14) we obtain

$$
\left(\chi_{R_{h}^{p}\left(\cdot, u_{N}(\nu) ; \nu\right)}^{X_{m}^{m}}, v_{N}\right)_{X_{h}^{m}}=\sum_{q=1}^{Q_{f}} \Theta_{q}^{f}(\nu) f_{q, h}^{p d}\left(v_{N}\right)-\sum_{j=1}^{N} \sum_{q=1}^{Q_{a}} u_{N}^{j}(\nu) \Theta_{q}^{a}(\nu) a_{q, h}^{p d}\left(\xi_{j}^{p}, v_{N}\right) .
$$


By superposition we recover

$$
\chi_{R_{h}^{p}\left(\cdot, u_{N}(\nu) ; \nu\right)}^{X_{m}^{m}}=\sum_{q=1}^{Q_{f}} \Theta_{q}^{f}(\nu) \chi_{f_{q, h}^{X_{h}^{m}(\cdot)}}^{X_{p}^{m}}-\sum_{j=1}^{N} \sum_{q=1}^{Q_{a}} u_{N}^{j}(\nu) \Theta_{q}^{a}(\nu) \chi_{a_{q, h}^{p d}\left(\xi_{j}^{p}, \cdot\right)}^{X_{h}^{m}} .
$$

Clearly, the terms boxed can be computed off-line. Each Riesz representation element needs the solution of a linear system of size $\mathcal{N}^{m}$.

Finally

$$
\begin{aligned}
& \left(\varepsilon_{N}^{p, m}(\nu)\right)^{2}=\sum_{q=1}^{Q_{f}} \sum_{\tilde{q}=1}^{Q_{f}} \Theta_{q}^{f}(\nu) \overline{\Theta_{\tilde{q}}^{f}(\nu)} \overline{\left(\chi_{f_{q, h}^{p d}(\cdot)}^{X_{h}^{m}}, \chi_{f_{\tilde{q}, h}^{p d}(\cdot)}^{X_{h}^{m}}\right)_{X_{h}^{m}}}+ \\
& \sum_{q=1}^{Q_{a}} \sum_{k=1}^{N} \sum_{\tilde{q}=1}^{Q_{a}} \sum_{\tilde{k}=1}^{N} u_{N}^{k}(\nu) \Theta_{q}^{a}(\nu) \overline{u_{N}^{\tilde{k}}(\nu)} \overline{\Theta_{\tilde{q}}^{a}(\nu)} \overline{\left(\chi_{a_{q, h}^{X_{h}^{m}}\left(\xi_{k}^{p}, \cdot\right)}^{X_{0}^{m}}, \chi_{a_{\tilde{q}, h}^{X_{h}^{m}}\left(\xi_{\tilde{k}}^{p}, \cdot\right)}^{Q_{0}^{m}} X_{h}^{m}\right.}-
\end{aligned}
$$

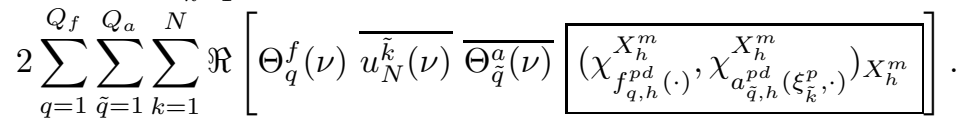

Here $\Re[\cdot]$ denotes the real part of a complex number. The quantities in the rectangles can be precomputed in the off-line part, once and for all. The number of operations in the on-line part is independent of the dimension $\mathcal{N}^{m}$ of the truth approximation spaces and is of order

$$
Q_{f}^{2}+Q_{a}^{2} N^{2}+Q_{f} Q_{a} N
$$

The computation of $\varepsilon_{M}^{d, m}(\nu)$ can be completed in a similar way.

5.3. Computing $\bar{\beta}^{p, m}(\nu), m \in\{p, p d\}$. The off-line construction of the lower bound of the inf-sup parameter remains the most expensive part of the algorithm, particularly for non-coercive problems with resonances such as Maxwell's equations. There are several algorithms to achieve this goal and we refer the readers to $[16,6]$ for the most recent successive constraint method (SCM). It finds, through a greedy algorithm, $K$ points $\nu_{1}, \ldots, \nu_{K}$ in the parameter domain $\mathcal{D}$. The exact inf-sup numbers at these $K$ points are found by solving the corresponding eigenvalue problems. Then, a rigorous lower bound $\bar{\beta}^{p, m}(\nu)$ for any $\nu \in \mathcal{D}$ is obtained by solving a local linear program. This on-line procedure is independent of the dimension of the truth approximation spaces ensuring a low computational cost for evaluating $\bar{\beta}^{p, m}(\nu)$. In the following, for the completeness of this paper, we describe SCM following [7] for a general affine bilinear form denoted by

$$
a^{\mathcal{N}}(w, v ; \nu) \equiv \sum_{q=1}^{Q} \Theta^{q}(\nu) a_{q}^{\mathcal{N}}(w, v), \quad \forall w, v \in X^{\mathcal{N}} .
$$

The methodology is defined for the coercive and then the non-coercive case.

5.3.1. Coercive case. The coercivity constant is

$$
\alpha^{\mathcal{N}}(\nu) \equiv \inf _{w \in X^{\mathcal{N}}} \frac{a^{\mathcal{N}}(w, w ; \nu)}{\|w\|_{X^{\mathcal{N}}}^{2}}=\inf _{w \in X^{\mathcal{N}}} \sum_{q=1}^{Q} \Theta^{q}(\nu) \frac{a_{q}^{\mathcal{N}}(w, w)}{\|w\|_{X^{\mathcal{N}}}^{2}}=\inf _{w \in X^{\mathcal{N}}} \sum_{q=1}^{Q} \Theta^{q}(\nu) y_{q}(w)
$$


Here, we set $y_{q}(w)=\frac{a_{q}^{\mathcal{N}}(w, w)}{\|w\|_{X \mathcal{N}}^{2}}$. Obviously, $\left(y_{1}(w), \ldots, y_{Q}(w)\right)$ belongs to the following set

$$
\mathcal{Y} \equiv\left\{y=\left(y_{1}, \ldots, y_{Q}\right) \in \mathbb{R}^{Q} \mid \exists w \in X^{\mathcal{N}} \text { s.t. } y_{q}=y_{q}(w), 1 \leq q \leq Q\right\} .
$$

Having defined the set $\mathcal{Y}$, our coercivity constant can be found by solving the following minimization problem:

$$
\alpha^{\mathcal{N}}(\nu)=\inf _{y \in \mathcal{Y}} \mathcal{J}(\nu ; y)
$$

where, the objective function $\mathcal{J}: \mathcal{D} \times \mathbb{R}^{Q} \rightarrow \mathbb{R}$ is defined as

$$
\mathcal{J}(\nu ; y)=\sum_{q=1}^{Q} \Theta^{q}(\nu) y_{q} .
$$

Problem (5.7) appears like a minimization problem of a linear functional over a compact subset of $\mathbb{R}^{Q}$.

We only need to characterize the set $\mathcal{Y}$ now. The idea of SCM is to build two sets $\mathcal{Y}_{L B}$ and $\mathcal{Y}_{U B}$ over which the minimization of $\mathcal{J}$ is feasible and which satisfies $\mathcal{Y}_{U B} \subset$ $\mathcal{Y} \subset \mathcal{Y}_{L B}$. Therefore, we can perform the minimization on these two sets to obtain an upper bound and a lower bound for $\alpha^{\mathcal{N}}(\nu)$. For this purpose, we define

$$
\sigma_{q}^{-} \equiv \inf _{w \in X^{\mathcal{N}}} y_{q}(w), \quad \sigma_{q}^{+} \equiv \sup _{w \in X^{\mathcal{N}}} y_{q}(w), \quad 1 \leq q \leq Q
$$

and let $\mathcal{B}_{Q} \equiv \Pi_{q=1}^{Q}\left[\sigma_{q}^{-}, \sigma_{q}^{+}\right] \subset \mathbb{R}^{Q}$. Obviously, $\mathcal{Y} \subset \mathcal{B}_{Q}$.

To properly define $\mathcal{Y}_{L B}$ and $\mathcal{Y}_{U B}$, we introduce two parameter sets $\Xi \equiv\left\{\nu_{1} \in\right.$ $\left.\mathcal{D}, \ldots, \nu_{J} \in \mathcal{D}\right\}$ and $C_{K} \equiv\left\{\nu_{1} \in \mathcal{D}, \ldots, \nu_{K} \in \mathcal{D}\right\} . \quad \Xi$ is a (rather large) sample set of grid points in the parameter domain (e.g. defined from a mesh) and $C_{K}$ is any subset of $\Xi$. Let $P_{M}(\nu ; E)$ denote the $M$ points closest to $\nu$ in $E$ with $E$ being $\Xi$ or $C_{K}$.

We are now ready to define $\mathcal{Y}_{L B}$ and $\mathcal{Y}_{U B}$ : For a given $C_{K}$ (and $M_{\alpha} \in \mathbb{N}, M_{+} \in \mathbb{N}$, and $\Xi$ ), we define

$$
\begin{aligned}
\mathcal{Y}_{L B}\left(\nu ; C_{K}\right) \equiv\left\{y \in \mathcal{B}_{Q} \mid\right. & \sum_{q=1}^{Q} \Theta^{q}\left(\nu^{\prime}\right) y_{q} \geq \alpha^{\mathcal{N}}\left(\nu^{\prime}\right), \forall \nu^{\prime} \in P_{M_{\alpha}}\left(\nu ; C_{K}\right) ; \\
& \left.\sum_{q=1}^{Q} \Theta^{q}\left(\nu^{\prime}\right) y_{q} \geq \alpha_{L B}\left(\nu^{\prime}, C_{K-1}\right), \forall \nu^{\prime} \in P_{M_{+}}\left(\nu ; \Xi \backslash C_{K}\right)\right\},
\end{aligned}
$$

and $\mathcal{Y}_{U B}\left(C_{K}\right) \equiv\left\{y^{*}\left(\nu_{k}\right), 1 \leq k \leq K\right\}$ for $y^{*}(\nu) \equiv \operatorname{argmin}_{y \in \mathcal{Y}} \mathcal{J}(\nu ; y)$. Define

$$
\alpha_{L B}\left(\nu, C_{0}\right) \equiv 0 ; \quad \alpha_{L B}\left(\nu ; C_{K}\right)=\inf _{y \in \mathcal{Y}_{L B}\left(\nu ; C_{K}\right)} \mathcal{J}(\nu ; y) \quad \text { for } K>0,
$$

and

$$
\alpha_{U B}\left(\nu ; C_{K}\right)=\inf _{y \in \mathcal{Y}_{U B}\left(C_{K}\right)} \mathcal{J}(\nu ; y)
$$


One can prove [7] that, for given $C_{K}$ (and $M_{\alpha} \in \mathbb{N}, M_{+} \in \mathbb{N}$, and $\left.\Xi\right), \alpha_{L B}\left(\nu ; C_{K}\right) \leq$ $\alpha^{\mathcal{N}}(\nu) \leq \alpha_{U B}\left(\nu ; C_{K}\right), \forall \nu \in \mathcal{D}$; and, for any $\nu \in \Xi, \alpha_{L B}\left(\nu, C_{K}\right)$ is nondecreasing, $\alpha_{U B}\left(\nu, C_{K}\right)$ nonincreasing and $\frac{\alpha_{U B}\left(\nu, C_{K}\right)-\alpha_{L B}\left(\nu, C_{K}\right)}{\alpha_{U B}\left(\nu, C_{K}\right)}$ nonincreasing as $K$ increases,

Note that (5.8), (5.9) is in fact a Linear Program (LP). LP (5.9) contains $Q$ design variables and $2 Q+M_{\alpha}+M_{+}$(one-sided) inequality constraints: the operation count for the on-line stage $\nu \rightarrow \alpha_{L B}(\nu)$ is independent of $\mathcal{N}$.

It only remains to determine $C_{K}$. It is constructed by an off-line "greedy" algorithm. Given $M_{\alpha} \in \mathbb{N}, M_{+} \in \mathbb{N}, \Xi$, and a tolerance $\epsilon_{\alpha} \in[0,1]$, the algorithm reads:

(1.) Set $K=1$ and choose $C_{1}=\left\{\nu_{1}\right\}$ arbitrarily.

(2.) Find $\nu_{K+1}=\operatorname{argmax}_{\nu \in \Xi} \frac{\alpha_{U B}\left(\nu ; C_{K}\right)-\alpha_{L B}\left(\nu ; C_{K}\right)}{\alpha_{U B}\left(\nu ; C_{K}\right)}$.

(3.) Update $C_{K+1}=C_{K} \cup\left\{\nu_{K+1}\right\}$.

(4.) Repeat (2) and (3) until $\max _{\nu \in \Xi} \frac{\alpha_{U B}\left(\nu ; C_{K_{\max }}\right)-\alpha_{L B}\left(\nu ; C_{K_{\max }}\right)}{\alpha_{U B}\left(\nu ; C_{K_{\max }}\right)} \leq \epsilon_{\alpha}$.

5.3.2. Non-coercive case. For the non-coercive case, we need to find a lower bound of the inf-sup constant,

$$
\beta^{\mathcal{N}}(\nu) \equiv \inf _{\omega \in X^{\mathcal{N}}} \sup _{v \in X^{\mathcal{N}}} \frac{\left|a^{\mathcal{N}}(\omega, v ; \nu)\right|}{\|\omega\|_{X^{\mathcal{N}}}\|v\|_{X^{\mathcal{N}}}} .
$$

If we define an operator $T^{\nu}: X^{\mathcal{N}} \rightarrow X^{\mathcal{N}}$ as $\left(T^{\nu} w, v\right)_{X^{\mathcal{N}}}=a^{\mathcal{N}}(w, v ; \nu), \forall v \in X^{\mathcal{N}}$, it follows that

$$
\beta^{\mathcal{N}}(\nu)=\inf _{w \in X^{\mathcal{N}}} \frac{\left\|T^{\nu} w\right\|_{X^{\mathcal{N}}}}{\|w\|_{X^{\mathcal{N}}}}
$$

such that

$$
\left(\beta^{\mathcal{N}}(\nu)\right)^{2}=\inf _{w \in X^{\mathcal{N}}} \frac{\left(T^{\nu} w, T^{\nu} w\right)_{X^{\mathcal{N}}}}{\|w\|_{X^{\mathcal{N}}}^{2}} .
$$

To expand it, we must define operators $T^{q}: X^{\mathcal{N}} \rightarrow X^{\mathcal{N}}$ as

$$
\left(T^{q} w, v\right)_{X^{\mathcal{N}}}=a_{q}^{\mathcal{N}}(w, v), \forall v \in X^{\mathcal{N}}, 1 \leq q \leq Q .
$$

Realizing $T^{\nu} w \equiv \sum_{q=1}^{Q} \Theta^{q}(\nu) T^{q} w$, we can expand $\left(\beta^{\mathcal{N}}(\nu)\right)^{2}$ as

$$
\begin{aligned}
\left(\beta^{\mathcal{N}}(\nu)\right)^{2} & =\inf _{w \in X^{\mathcal{N}}} \sum_{q^{\prime}=1}^{Q} \sum_{q^{\prime \prime}=1}^{Q} Z_{q^{\prime \prime}}^{q^{\prime}}(\nu) \frac{\left(T^{q^{\prime}} w, T^{q^{\prime \prime}} w\right)_{X^{\mathcal{N}}}}{\|w\|_{X^{\mathcal{N}}}^{2}} \\
& =\inf _{w \in X^{\mathcal{N}}} \sum_{q^{\prime}=1}^{Q} \sum_{q^{\prime \prime}=q^{\prime}}^{Q} \frac{Z_{q^{\prime \prime}}^{q^{\prime}}(\nu)}{1+\delta_{q^{\prime} q^{\prime \prime}}} \frac{\left(T^{q^{\prime}} w, T^{q^{\prime \prime}} w\right)_{X^{\mathcal{N}}}+\left(T^{q^{\prime \prime}} w, T^{q^{\prime}} w\right)_{X^{\mathcal{N}}}}{\|w\|_{X^{\mathcal{N}}}^{2}} .
\end{aligned}
$$

Here, $Z_{q^{\prime \prime}}^{q^{\prime}}(\nu)=\Theta^{q^{\prime}}(\nu) \Theta^{q^{\prime \prime}}(\nu)$ and $\delta_{q^{\prime} q^{\prime \prime}}$ is the Kronecker delta. Next, we identify

$$
\begin{gathered}
\frac{Z_{q^{\prime \prime}}^{q^{\prime}}(\nu)}{1+\delta_{q^{\prime} q^{\prime \prime}}}, 1 \leq q^{\prime} \leq q^{\prime \prime} \leq Q \longmapsto \widehat{\Theta}^{q}(\nu), 1 \leq q \leq \widehat{Q} \equiv \frac{Q(Q+1)}{2}, \\
\left(T^{q^{\prime}} w, T^{q^{\prime \prime}} w\right)_{X \mathcal{N}}+\left(T^{q^{\prime \prime}} w, T^{q^{\prime}} w\right)_{X \mathcal{N}}, 1 \leq q^{\prime} \leq q^{\prime \prime} \leq Q \longmapsto \widehat{a}_{q}^{\mathcal{N}}(w, v), 1 \leq q \leq \widehat{Q},
\end{gathered}
$$


and obtain

$$
\left(\beta^{\mathcal{N}}(\nu)\right)^{2} \equiv \inf _{w \in X^{\mathcal{N}}} \sum_{q=1}^{\widehat{Q}} \widehat{\Theta}^{q}(\nu) \frac{\widehat{a}_{q}^{\mathcal{N}}(w, w)}{\|w\|_{X^{\mathcal{N}}}^{2}} .
$$

Hence $\left(\beta^{\mathcal{N}}(\nu)\right)^{2}$ can be interpreted as the coercivity constant for the bilinear form

$$
\widehat{\alpha}^{\mathcal{N}}(\nu) \equiv \inf _{w \in X^{\mathcal{N}}} \sum_{q=1}^{\widehat{Q}} \widehat{\Theta}^{q}(\nu) \frac{\widehat{a}_{q}^{\mathcal{N}}(w, w)}{\|w\|_{X^{\mathcal{N}}}^{2}} .
$$

We may then directly apply the SCM procedure defined above to (5.12).

Before extending this to the complex case, we interpret the expansion above in terms of matrices: if we let $\boldsymbol{w}$ denote the vector of degrees of freedom for $w \in X^{\mathcal{N}}$ and $M_{T^{q^{\prime}}, T^{q^{\prime \prime}}}$ denote the matrix corresponding to $\left(T^{q^{\prime}} w, T^{q^{\prime \prime}} w\right)_{X^{\mathcal{N}}}$, we rewrite (5.11) as

$$
\left(\beta^{\mathcal{N}}(\nu)\right)^{2}=\sum_{q^{\prime}=1}^{Q} \sum_{q^{\prime \prime}=q^{\prime}}^{Q} \frac{Z_{q^{\prime \prime}}^{q^{\prime}}(\nu)}{1+\delta_{q^{\prime} q^{\prime \prime}}} \frac{\boldsymbol{w}^{T}\left(M_{T^{q^{\prime}}, T^{q^{\prime \prime}}}+M_{T^{q^{\prime \prime}}, T^{q^{\prime}}}\right) \boldsymbol{w}}{\|w\|_{X^{\mathcal{N}}}^{2}} .
$$

When $\Theta^{q}(\nu)$ is complex, we have

$$
\begin{aligned}
\left(\beta^{\mathcal{N}}(\nu)\right)^{2}= & \inf _{w \in X_{\mathcal{N}}} \sum_{q^{\prime}=1}^{Q} \sum_{q^{\prime \prime}=1}^{Q} Z_{q^{\prime \prime}}^{q^{\prime}}(\nu) \frac{\boldsymbol{w}^{H} M_{T^{q^{\prime}}, T^{\prime \prime}} \boldsymbol{w}}{\|w\|_{X^{\mathcal{N}}}^{2}} \\
= & \inf _{w \in X_{\mathcal{N}}} \sum_{q=1}^{Q} Z_{q}^{q}(\nu) \frac{\boldsymbol{w}^{H} M_{T^{q}, T^{q}} \boldsymbol{w}}{\|w\|_{X^{\mathcal{N}}}^{2}} \\
& \quad+\sum_{q^{\prime}=1}^{Q} \sum_{q^{\prime \prime}=q^{\prime}+1}^{Q} \frac{Z_{q^{\prime \prime}}^{q^{\prime}}(\nu) \boldsymbol{w}^{H} M_{T^{q^{\prime}}, T^{q^{\prime \prime}}} \boldsymbol{w}+Z_{q^{\prime}}^{q^{\prime \prime}}(\nu) \boldsymbol{w}^{H} M_{T^{q^{\prime \prime}}, T q^{\prime}} \boldsymbol{w}}{\|w\|_{X^{\mathcal{N}}}^{2}} \\
= & \inf _{w \in X_{\mathcal{N}}} \sum_{q=1}^{Q} Z_{q}^{q}(\nu) \frac{\boldsymbol{w}^{H} M_{T^{q}, T^{q}} \boldsymbol{w}}{\|w\|_{X^{\mathcal{N}}}^{2}} \\
& \quad+\sum_{q^{\prime}=1}^{Q} \sum_{q^{\prime \prime}=q^{\prime}+1}^{Q} \frac{\boldsymbol{w}^{H}\left(Z_{q^{\prime \prime}}^{q^{\prime}}(\nu) M_{T^{q^{\prime}}, T^{q^{\prime \prime}}}+Z_{q^{\prime}}^{q^{\prime \prime}}(\nu) M_{T^{q^{\prime}}, T^{q^{\prime \prime}}} T\right) \boldsymbol{w}}{\|w\|_{X^{\mathcal{N}}}^{2}} .
\end{aligned}
$$

Here, $Z_{q^{\prime \prime}}^{q^{\prime}}(\nu)=\Theta^{q^{\prime}}(\nu) \bar{\Theta}^{q^{\prime \prime}}(\nu)=\bar{Z}_{q^{\prime}}^{q^{\prime \prime}}(\nu)$.

Note that, when $z$ is a complex number, $X$ a complex vector and $A$ a real matrix, we have

$$
\begin{aligned}
& X^{H}\left(z A+\bar{z} A^{T}\right) X=2 \Re z\left(\Re X^{T} \Im X^{T}\right)\left(\begin{array}{cc}
A & 0 \\
0 & A
\end{array}\right)\left(\begin{array}{c}
\Re X \\
\Im X
\end{array}\right) \\
&+2 \Im z\left(\Re X^{T} \Im X^{T}\right)\left(\begin{array}{cc}
0 & -A \\
A & 0
\end{array}\right)\left(\begin{array}{c}
\Re X \\
\Im X
\end{array}\right) \\
&=2 \Re z\left(\Re X^{T} \Im X^{T}\right)\left(\begin{array}{cc}
\frac{A+A^{T}}{2} & 0 \\
0 & \frac{A+A^{T}}{2}
\end{array}\right)\left(\begin{array}{c}
\Re X \\
\Im X
\end{array}\right) \\
&+2 \Im z\left(\Re X^{T} \Im X^{T}\right)\left(\begin{array}{cc}
0 & \frac{A^{T}-A}{2} \\
\frac{A-A^{T}}{2} & 0
\end{array}\right)\left(\begin{array}{c}
\Re X \\
\Im X
\end{array}\right) .
\end{aligned}
$$




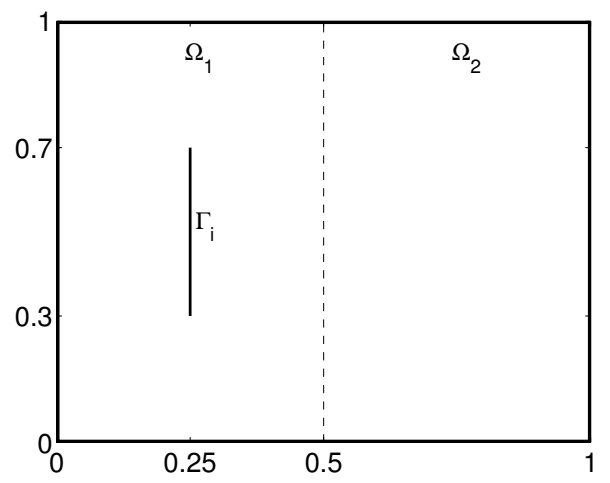

FIG. 6.1. Sketch of the geometry of the electromagnetic cavity problem.

Here, $\Re$ and $\Im$ indicate real and imaginary parts, respectively, ${ }^{H}$ means conjugate transpose and.$^{T}$ transpose. This allows us to obtain an expansion as in the real case.

6. Numerical examples. After having laid out the theoretical foundation and the computational implications of the proposed methods, we now demonstrate the validity and efficiency on the following cavity problem associated with the solution of Maxwell's equations. We consider the two-dimensional Maxwell's equations on TEform, that is, we assume in (2.1) that $\boldsymbol{E}(x, y)=\left(E_{x}, E_{y}, 0\right)$ and $\boldsymbol{H}(x, y)=\left(0,0, H_{z}\right)$. We seek the solution to this problem in the closed geometry, illustrated in Fig. 6.1 where we assume all exterior boundary to be perfectly electrically conducting with vanishing tangential electric fields. The cavity is loaded with two materials, each occupying half of the cavity. For simplicity we assume that $\Omega_{1}$ of the cavity is vacuum filled in which case $\varepsilon_{1}=\mu_{1}=1$ while the material parameters in $\Omega_{2}$ are the parameters of the problem, that is, $\nu=\left(\varepsilon_{2}, \mu_{2}\right)$.

The equivalent equation in second order form, (2.2), is solved using the discontinuous Galerkin method discussed in Sec. 3 and the spaces $X_{h}^{m}(m \in\{p, d, p d\})$ containing piecewise polynomial of degree 4 on the meshes shown in Figure 6.2.

As a source, we consider a simple dipole antenna, modeled as a current

$$
J_{x}=0, \quad\left(J_{y}, v\right)=\int_{\Gamma_{i}} \cos \left(\omega\left(y-\frac{1}{2}\right)\right) v d s
$$

where $\Gamma_{i}$ reflects the antenna. We use $\omega=5 \pi / 2$.

Without any special significance, we choose the functional of interest as

$$
s(\boldsymbol{E})=\int_{\Omega_{2}} E_{x}+E_{y} d \boldsymbol{x} .
$$

We consider numerical results for $\epsilon_{2} \in[2,6], \mu_{2} \in[1.0,1.2]$, same settings as the twoparameter case in [7], and use a Cartesian grid of $512 \times 33$ as the set $\mathcal{S}$. The lower bound $\bar{\beta}^{p, p}(\nu)$ is captured by the SCM, see Figure 6.3. Recall that we assume that the inf-sup numbers (4.3) are uniformly bounded away from zero for the problems to be well defined. However, in Figure 6.3, there are 12 visible bands in which the lower bound is below $10^{-6}$. As discussed earlier in Sec. 4.1, this indicates that the assumption on the inf-sup number is violated for these parameters. We see that, 
FiG. 6.2. The three grids used in the computational example, defining $X_{h}^{p}, X_{h}^{d}$, and $X_{h}^{p d}$, respectively
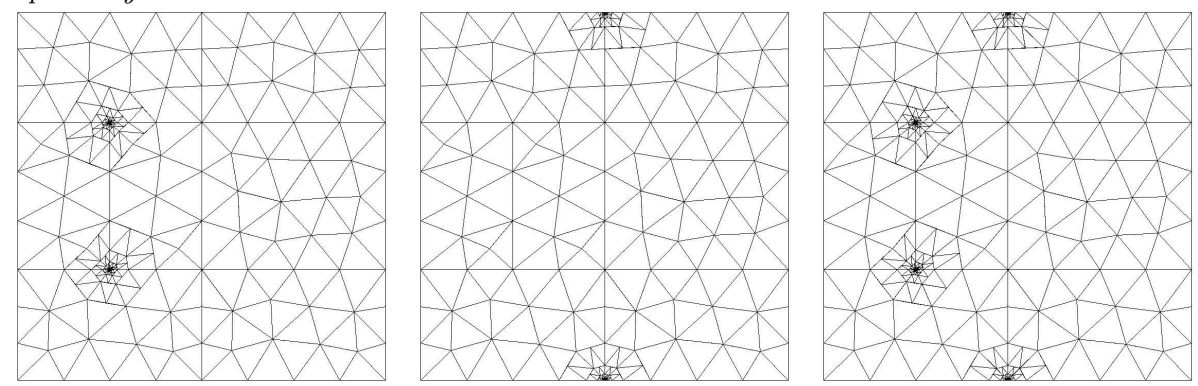

FiG. 6.3. Contour plot of $\log _{10} \bar{\beta}^{p, p}(\nu)^{2}$ computed by the SCM.

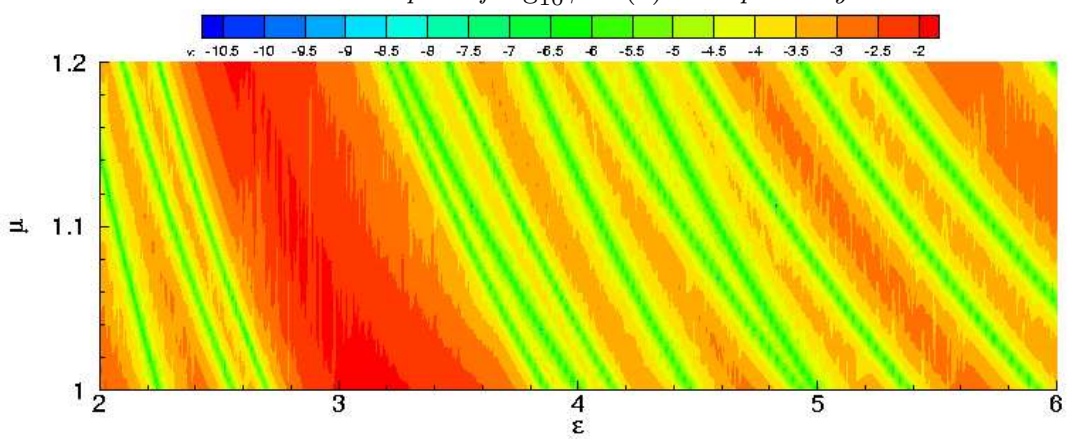

through this lower bound computation by SCM, we can identify such parameters and exclude them from our reduced basis calculations.

We take $N=M=50$ and build $X_{N}^{p}$ and $X_{M}^{d}$. See Figure 6.4 for the sets $S_{N}^{p}=$ $\left\{\nu_{1}^{p}, \ldots, \nu_{50}^{p}\right\}$ and $S_{M}^{d}=\left\{\nu_{1}^{d}, \ldots, \nu_{50}^{d}\right\}$ chosen by the greedy algorithm.

FIG. 6.4. The points selected by the RBM to build the bases for the primal (top) and dual (bottom) problems using the error estimate for the solutions.

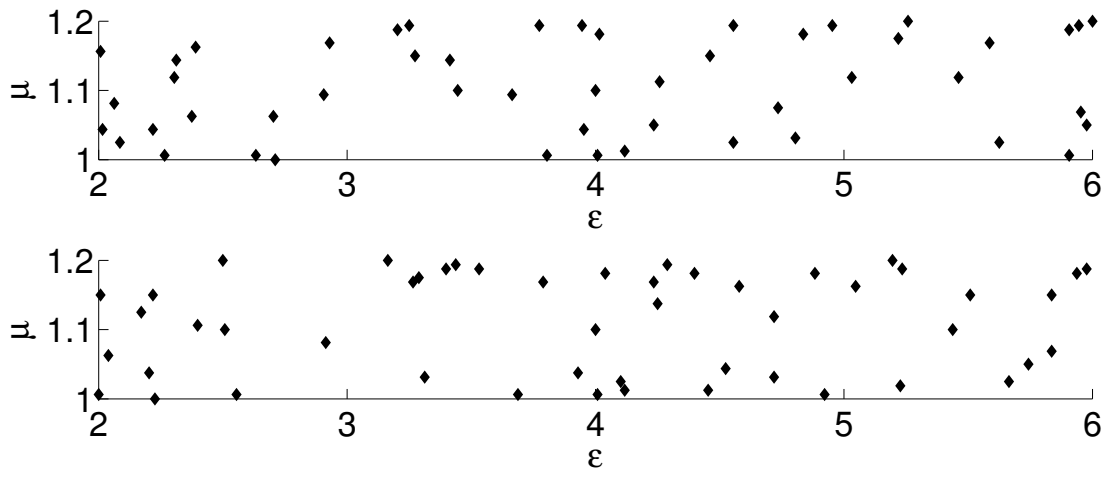

We use these two spaces to compute the reduced basis solution for any parameter in $[2,6] \times[1,1.2]$. See Figure 6.5 for sample (purely imaginary) primal and (real) dual solutions at $\left(\epsilon_{2}, \mu_{2}\right)=(2,1)$. We observe discontinuity and strong singularity 
TABLE 6.1

The error (the $H$ (curl) difference from the truth approximation) and time (the on-line computation time relative to that of the truth approximation) for $R B$ calculation with respect to number of bases.

Primal Problem

\begin{tabular}{|c|c|c|}
\hline $\mathrm{N}$ & error & time \\
\hline 10 & $9.5 \mathrm{e}-1$ & $2.1 \mathrm{e}-4$ \\
20 & $3.2 \mathrm{e}-2$ & $4.2 \mathrm{e}-4$ \\
30 & $2.6 \mathrm{e}-5$ & $6.4 \mathrm{e}-4$ \\
\hline
\end{tabular}

Dual Problem

\begin{tabular}{|c|c|c|}
\hline $\mathrm{M}$ & error & time \\
\hline 10 & $1.2 \mathrm{e}-1$ & $2.1 \mathrm{e}-4$ \\
20 & $4.0 \mathrm{e}-3$ & $4.2 \mathrm{e}-4$ \\
30 & $5.0 \mathrm{e}-5$ & $6.4 \mathrm{e}-4$ \\
\hline
\end{tabular}

FIG. 6.5. Sample truth approximations: the first row shows the (purely imaginary) solution to the primal problem, on the second row is the (real) solution to the dual problem. This essential differences highlights the value of having different meshes for the primal and dual problems.

$\Im E_{x}$
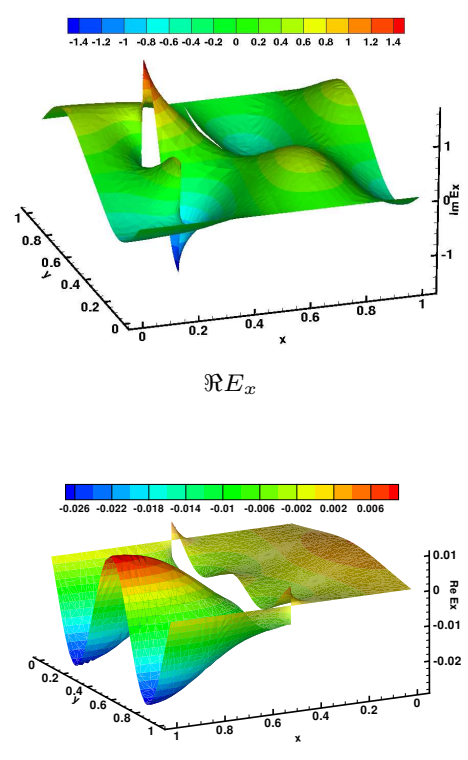

$\Im E_{y}$
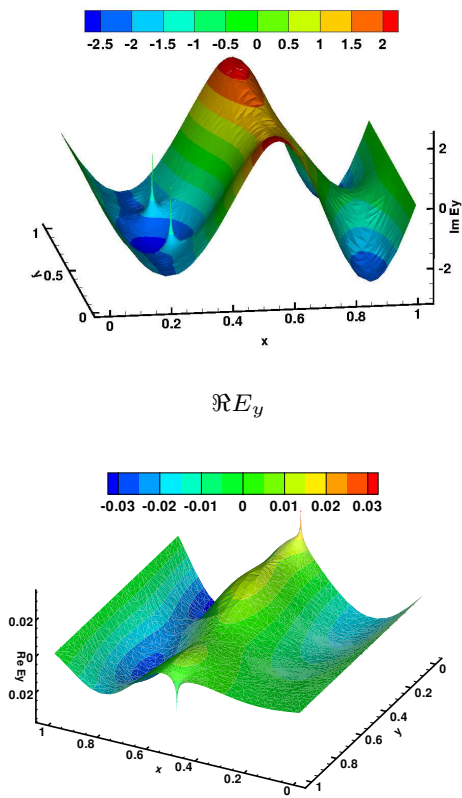

in the primal and dual solutions. These make it challenging for the RBM to perform well. However, as we will show later, our method can provide accurate reduced basis solution that is converging exponentially. In table 6.1, we show the $\mathrm{H}$ (curl) error and relative computation time of the RB solutions with the dimension of the RBM spaces being 10, 20 and 30 . We observe that, as we increase the dimension of the RBM space, the RB solution converges to the truth approximation in the $H$ (curl) norm. We also see that, in the last column, the relative CPU time to obtain these RB approximations is essentially negligible compared to that of the truth approximation.

Next, we test our error estimate on a set of 1300 points away from the resonance lines in the parameter domain, see Figure 6.6 for the set, $\Xi_{\mathrm{t}, 1} \subset \mathcal{S} \backslash\left(S_{N}^{p} \cup S_{M}^{d}\right)$. We compute, for any $\nu \in \Xi_{\mathrm{t}, 1}$, the truth approximation and the reduced basis primal/dual 
FIG. 6.6. $\Xi_{\mathrm{t}, 1}$ contains 1300 points.

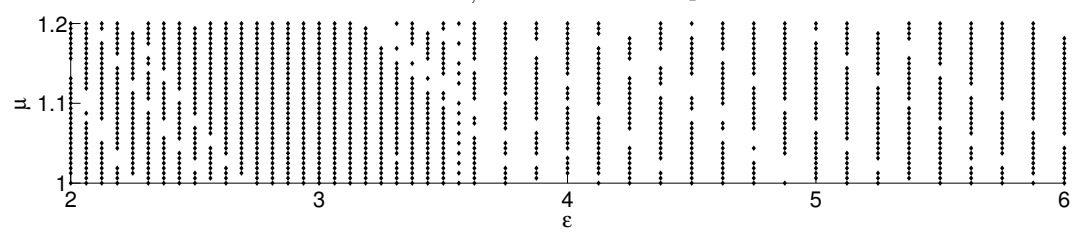

solutions for $N=10, \ldots, 30$ and evaluate the H(curl) norms of the errors $u_{h}^{p}(\nu)-$ $u_{N}(\nu)$ and $\psi_{h}^{d}(\nu)-\psi_{M}(\nu)$, the error estimates $\Delta_{N}^{p, p}$ and $\Delta_{M}^{d, d}$. In the first two rows of Figure 6.7, we plot the maximum, median, and minimum of these values over $\Xi_{\mathrm{t}, 1}$ and observe that all basically decrease exponentially with respect to $N$.

Finally, we sort $\Xi_{\mathrm{t}, 1}$ according to the corresponding lower bound and let $\Xi_{\mathrm{t}, 1}^{k}$ be the set of the first $k$ points in $\Xi_{\mathrm{t}, 1}$ for $k=1, \ldots, 1300$. The last row of Figures 6.7 represents the the maximum effectivity indices over $\Xi_{\mathrm{t}, 1}^{k}$ versus $k$ for reduced basis spaces of dimension $10,15,20,25$. We see that the error estimate is in general sharp and particularly sharp when $k$ is small, that is, when the parameter stays far away from the resonances. The error estimates deteriorate as we get closer to the resonances, but they are still legitimate convergence indicators. It is interesting, however, to note that the effectivity indices are essentially independent of the size of the reduced basis that is used for the computation. That is, the effectivity indices remain of the same magnitude when the errors becomes many magnitudes smaller as we increase the dimension of the reduced basis space. To show that our result above is not a coincidence of the fact $\Xi_{\mathrm{t}, 1} \subset \mathcal{S}$, we perform the same test on a set, $\Xi_{\mathrm{t}, 2}$, that contains 1000 centers of the rectangle cells of $\mathcal{S}$. See Figure 6.8 for $\Xi_{\mathrm{t}, 2}$. Note that, for $\Xi_{\mathrm{t}, 2}$, we exclude more points in the neighborhood of the resonance lines. This is a consequence of the SCM, which provides more conservative lower bound for $\nu \notin \mathcal{S}$ than for $\nu \in \mathcal{S}$. Thus, more points are considered to be "too close" to resonance and excluded from the computation. Addressing this shortfall of SCM is one of our ongoing works. Figure 6.9 shows that our method performs equally well on the set $\Xi_{\mathrm{t}, 2}$. We conclude that the method provides a reliable a posteriori estimator for both primal and dual problems.

Recall that the quantity of our interest is the output. There are two ways to compute the output. One is done similarly to Eq (4.7) where only the primal or dual solution is used, that is, $S_{N}(\nu)=l_{h}^{p d}\left(u_{N}(\nu)\right)$. The other is by Eq (4.13) where both the primal and dual solutions are included. Figure 6.10 shows the histories of convergence. We see that the latter converges much faster. In fact, the rate is quadratic with respect to that of the error of the solutions [22].

7. Concluding remarks. Certified reduced basis methods for the harmonic Maxwell's equations are developed. We examine several essential ingredients such as the a posteriori error estimates for the solution and output, off-line/on-line computation procedure, two different greedy algorithms to build the reduced basis spaces. We have applied the method to a challenging electromagnetic cavity problem. The rigor and high efficiency of the method are confirmed by the numerical results. Exponential convergence of the reduced basis approximation to the truth finite element approximation is observed. The reduced basis output also converges exponentially. Future work includes efficient extension to many-parameter problems, and investigation of reduced basis element method [24] for electromagnetics. 
Fig. 6.7. Results for the primal (left column) and dual (right column) problems on $\Xi_{\mathrm{t}, 1}$ : Plotted on the first row is the dimensions of the reduced basis space versus the H(curl) norm of the errors of the solution. The second row is the dimensions of the reduced basis space versus the H(curl) error estimate. The last row is the size of the test set versus the maximum effectivity index of the error estimates.
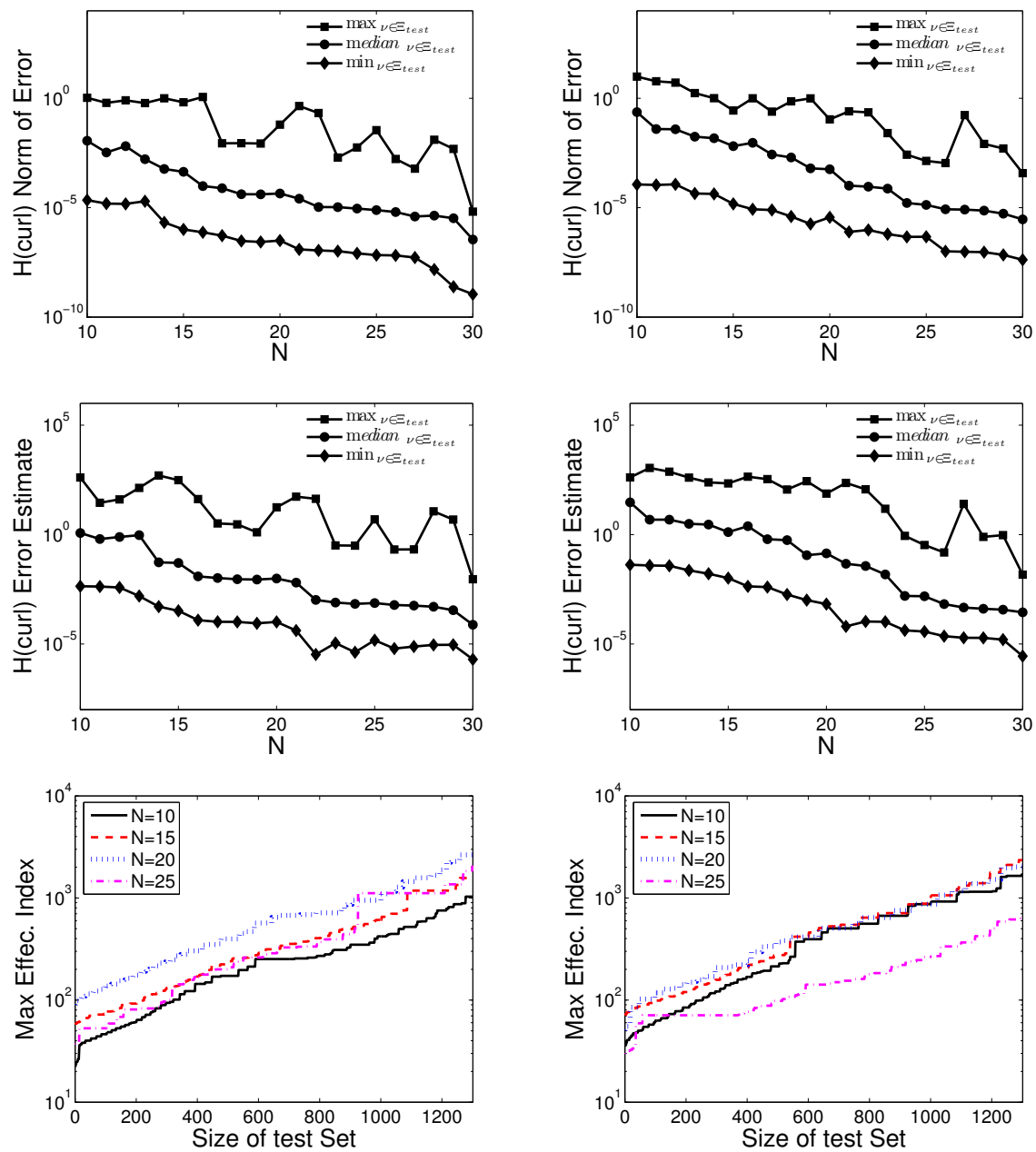

Acknowledgments. This work was partially supported by AFOSR Grant FA955007-1-0425. The authors would like to thank the anonymous referees for many constructive comments that lead to a better presentation of the paper.

\section{REFERENCES}

[1] B. O. Almroth, P. Stern, and F. A. Brogan. Automatic choice of global shape functions in structural analysis. AIAA Journal, 16:525-528, May 1978.

[2] D. N. Arnold, F. Brezzi, B. Cockburn, and L. D. Marini. Unified Analysis of discontinuous Galerkin methods for elliptic problems. SIAM J. Numer. Anal., 39(2002), pp. 1749-1779.

[3] E. Balmes. Parametric families of reduced finite element models: Theory and applications. Machanical Systems and Signal Processing, 10(4):381-394, 1996.

[4] M. Barrault, N. C. Nguyen, Y. Maday, and A. T. Patera. An "empirical interpolation" method: 
FIG. 6.8. $\Xi_{\mathrm{t}, 2}$ contains 1000 points.

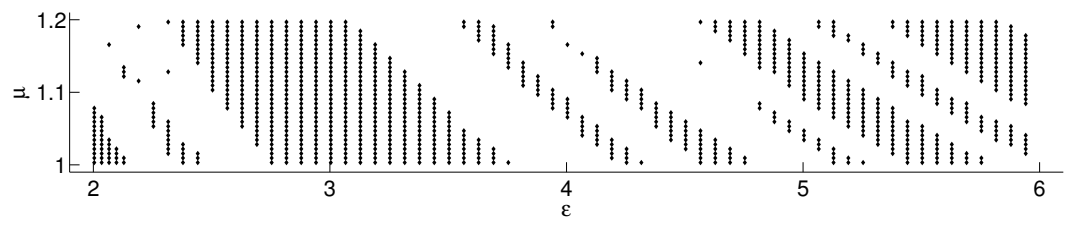

Application to efficient reduced-basis discretization of partial differential equations. $C$. $R$. Acad. Sci. Paris, Série I, 339:667-672, 2004.

[5] A. Barrett and G. Reddien. On the reduced basis method. Z. Angew. Math. Mech., 75(7):543$549,1995$.

[6] Y. Chen, J. S. Hesthaven, Y. Maday, and J. Rodríguez. A monotonic evaluation of lower bounds for inf-sup stability constants in the frame of reduced basis approximations. C. R. Acad. Sci. Paris, Ser. I, 346:1295-1300, 2008.

[7] Y. Chen, J. S. Hesthaven, Y. Maday, and J. Rodríguez. Improved successive constraint method based a posteriori error estimate for reduced basis approximation of $2 \mathrm{~d}$ maxwell's problem. $M 2 A N$, To Appear.

[8] J. P. Fink and W. C. Rheinboldt. On the error behavior of the reduced basis technique for nonlinear finite element approximations. Z. Angew. Math. Mech., 63(1):21-28, 1983.

[9] M. A. Grepl. Reduced-Basis Approximation and A Posteriori Error Estimation for Parabolic Partial Differential Equations. PhD thesis, MIT, June 2005.

[10] M. A. Grepl, Y. Maday, N. C. Nguyen, and A. T. Patera. Efficient reduced-basis treatment of nonaffine and nonlinear partial differential equations. Mathematical Modelling and Numerical Analysis, 41(3):575-605, 2007.

[11] P. Guillaume and M. Masmoudi. Solution to the time-harmonic Maxwell's equations in a waveguide; use of high order derivatives for solving the discrete problem. SIAM J. Numer. Anal., 34:1306-1330,1997.

[12] M. D. Gunzburger. Finite element methods for viscous incompressible flows. Computer Science and Scientific Computing. Academic Press Inc., Boston, MA, 1989. A guide to theory, practice, and algorithms.

[13] J.S. Hesthaven and T. Warburton. Nodal Discontinuous Galerkin Methods: Algorithms, Analysis, and Applications, volume 54 of Text in Applied Mathematics. Springer Verlag, New York, 2008.

[14] P. Houston, I. Perugia, A. Schneebeli, D. Schötzau. Interior penalty method for the indefinite time-harmonic Maxwell equations. Numer. Math. 100:485 - 518, 2005.

[15] P. Houston, I. Perugia, D. Schötzau. Mixed discontinuous Galerkin approximation of the Maxwell operator. SIAM J. Numer. Anal. 42:434 - 459, 2004.

[16] D.B.P. Huynh, G. Rozza, S. Sen, and A.T. Patera. A successive constraint linear optimization method for lower bounds of parametric coercivity and inf-sup stability constants. $C$. $R$. Acad. Sci. Paris, Série I., 345:473 - 478, 2007.

[17] K. Ito and S. S. Ravindran. A reduced basis method for simulation and control of fluid flows. Journal of Computational Physics, 143(2):403-425, July 1998.

[18] M.A. Jabbar, A.B. Azeman. Fast optimization of electromagnetic-problems: the reduced-basis finite element approach. IEEE Trans Magn, 40(4):2161-2163, 2004.

[19] M.A. Jabbar, A.B. Azeman. Multi-variable torque optimization for small spindle motors based on reduced-basis finite element formulation. International Conference on Power Electronics, Machines and Drives, 269-274, 2002.

[20] E.F. Knott, J.F. Shaeffer, M.T. Tuley. Radar cross section. SciTech Publishing, Inc., 2004.

[21] L. Machiels, Y. Maday, I. B. Oliveira, A. T. Patera, and D. V. Rovas. Output bounds for reduced-basis approximations of symmetric positive definite eigenvalue problems. C. $R$. Acad. Sci. Paris Sér. I Math., 331(2):153-158, 2000.

[22] Y. Maday, A. T. Patera, and D. V. Rovas. A blackbox reduced-basis output bound method for noncoercive linear problems. In Nonlinear partial differential equations and their applications. Collège de France Seminar, Vol. XIV (Paris, 1997/1998), volume 31 of Stud. Math. Appl., pages 533-569. North-Holland, Amsterdam, 2002.

[23] Y. Maday, A. T. Patera, and G. Turinici. Global a priori convergence theory for reducedbasis approximations of single-parameter symmetric coercive elliptic partial differential equations. C. R. Acad. Sci. Paris, Ser. I, 335:289-294, 2002. 
FIG. 6.9. Results for the primal (left column) and dual (right column) problems on $\Xi_{\mathrm{t}, 2}$ : Plotted on the first row is the dimensions of the reduced basis space versus the H(curl) norm of the errors of the solution. The second row is the dimensions of the reduced basis space versus the H(curl) error estimate. The last row is the size of the test set versus the maximum effectivity index of the error estimates.
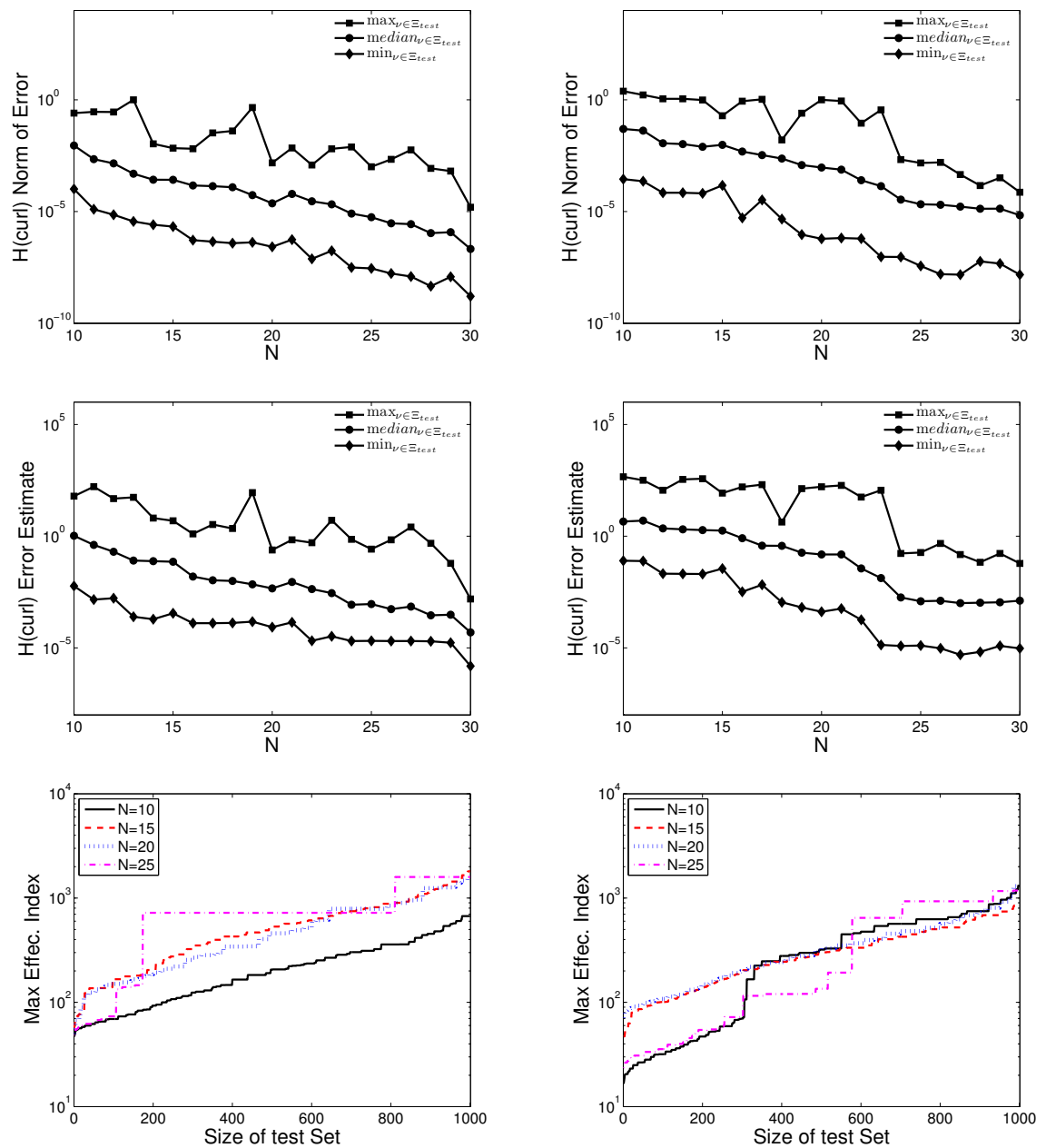

[24] Y. Maday, E. M. Rønquist, A reduced basis element method. J. Sci. Comput., 17(2002), 447 459.

[25] P. Monk. Finite Element Methods for Maxwell's Equations. Numerical Mathematics and Scientific Computing. Oxford University Press, Oxford, United Kingdom, 2003.

[26] N. C. Nguyen. Reduced-Basis Approximations and A Posteriori Error Bounds for Nonaffine and Nonlinear Partial Differential Equations: Application to Inverse Analysis. $\mathrm{PhD}$ thesis, Singapore-MIT Alliance, June 2005.

[27] A. K. Noor and J. M. Peters. Reduced basis technique for nonlinear analysis of structures. AIAA Journal, 45(172):487-496, April 1980.

[28] J. S. Peterson. The reduced basis method for incompressible viscous flow calculations. SIAM Journal on Scientific and Statistical Computing, 10(4):777-786, 1989.

[29] N. A. Pierce and M. B. Giles. Adjoint recovery of superconvergent functionals from PDE approximations. SIAM Review, 42(2):247-264, 2000.

[30] T. A. Porsching. Estimation of the error in the reduced basis method solution of nonlinear equations. Math. Comp., 45(172):487-496, 1985. 
FiG. 6.10. Comparison of histories of convergence for the two ways to compute the output.
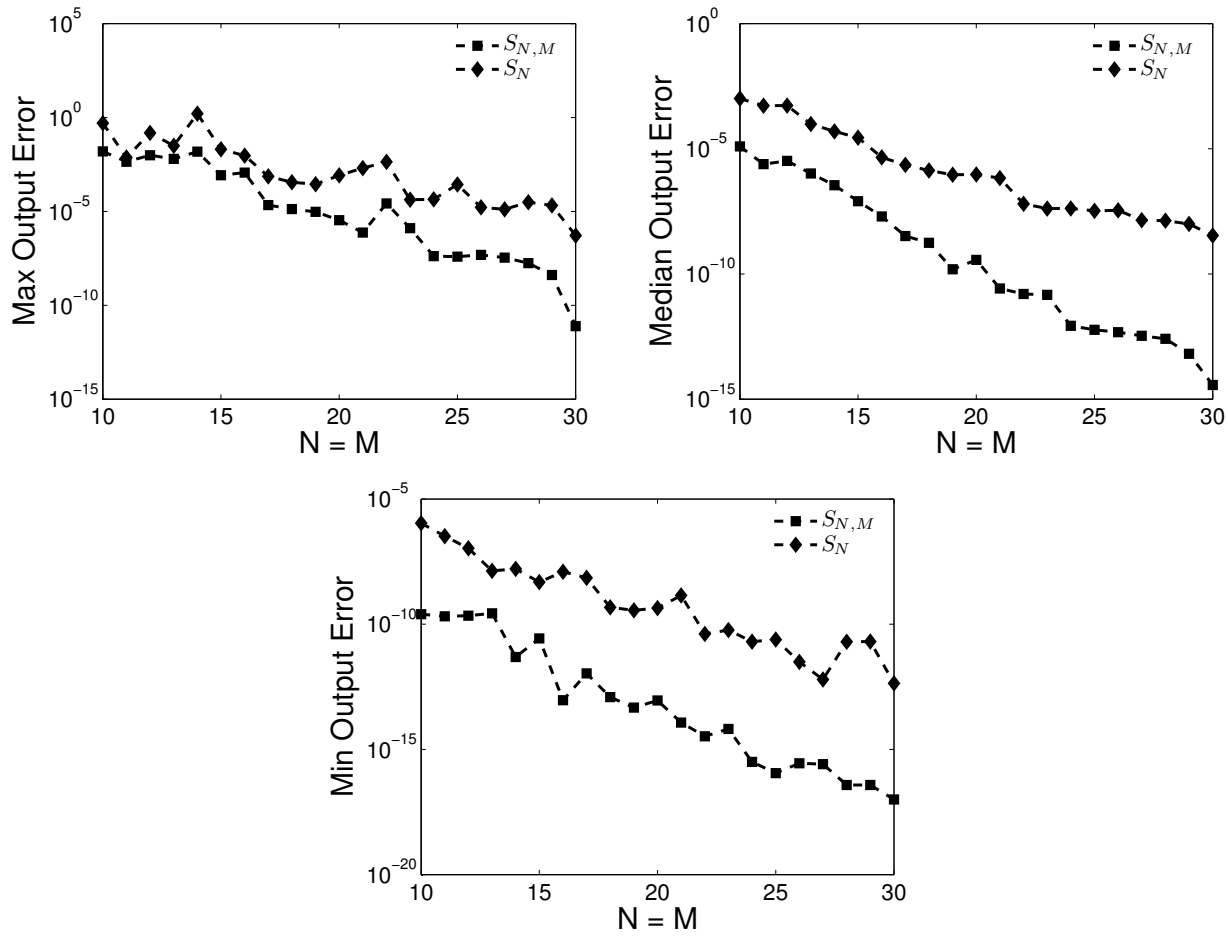

[31] C. Prud'homme, D. Rovas, K. Veroy, Y. Maday, A. T. Patera, and G. Turinici. Reliable realtime solution of parametrized partial differential equations: Reduced-basis output bound methods. Journal of Fluids Engineering, 124(1):70-80, March 2002.

[32] W. C. Rheinboldt. On the theory and error estimation of the reduced basis method for multiparameter problems. Nonlinear Anal., 21(11):849-858, 1993.

[33] D. Rovas. Reduced-Basis Output Bound Methods for Parametrized Partial Differential Equations. PhD Thesis, MIT, February 2003.

[34] G. Rozza. Shape Design by optimal flow control and reduced basis techniques: Applications to bypass configurations in haemodynamics. PhD thesis, EPFLNovember 2005.

[35] G. Rozza, D.B.P. Huynh, And A.T. PAtera. Reduced basis approximation and a posteriori error estimation for affinely parametrized elliptic coercive partial differential equations: Application to transport and continuum mechanics. Arch Comput Methods Eng, 15(3):229275,2008 .

[36] S. Sen. Reduced Basis Approximation and A Posteriori Error Estimation for Non-Coercive Elliptic Problems: Application to Acoustics. PhD thesis, MIT, June 2007.

[37] K. Veroy. Reduced-Basis Methods Applied to Problems in Elasticity: Analysis and Applications. PhD thesis, MIT, June 2003.

[38] K. Veroy, C. Prud'homme, and A. T. Patera. Reduced-basis approximation of the viscous burgers equation: Rigorous a posteriori error bounds. C. R. Acad. Sci. Paris, Série I, 337(9):619-624, November 2003.

[39] K. Veroy, C. Prud'homme, D.V. Rovas, and A.T.Patera. A posteriori error bounds for reducedbasis approximation of parametrized noncoercive and nonlinear elliptic partial differential equations. AIAA, 2003. 